\title{
OPTIC ISSUES IN ONGOING ERL PROJECTS
}

\section{S.L. Smith, B.D. Muratori, H.L. Owen,}

CCLRC Daresbury Laboratory

\author{
Keckwick Lane, Daresbury, Warrington WA4 4AD, UK
}

\section{G.H. Hoffstaetter}

Cornell University, Ithaca, New York

\section{V.N. Litvinenko,I. Ben-Zvi, M. Bai, J. Beebe-Wang, M. Blaskiewicz, J.M. Brennan,} R.Calaga, W. Fischer, X.Y. Chang, D. Kayran, J. Kewisch, W.W. MacKay, C. Montag, B.Parker,V.Ptitsyn, T. Roser, A. Ruggiero, T. Satogata, B. Surrow, S. Tepikian, D. Trbojevic, V. Yakimenko, S.Y. Zhang BNL, Upton, NY 11973, U.S.A.

\section{Ph. Piot}

Femilab, PO Box 500, Batavia IL 60510 , USA

Corresponding author: Susan Smith

CCLRC Daresbury Laboratory

Warrington WA4 4AD, UK

Phone: $+44(0) 1925603588$

FAX: $+44(0) 1925603124$

e-mail: $\underline{\text { s.1.smith@dl.ac.uk }}$

\begin{abstract}
A wide range of optics issues for Energy Recovery Linac (ERL) based projects are illustrated through the presentation of ongoing projects covering both light sources, at Cornell and Daresbury and high energy and nuclear physics accelerators at the Brookhaven National Laboratory. This presented range of projects demonstrates how the different design teams
\end{abstract}


see the challenges of studying and solving optics issues for their particular project's ERLs, with studies appropriate to the stage of maturity of the project. Finally as an illustration of the complexity and detail behind a single aspect of ERL optics design we present an overview of the highly important generic topic of longitudinal phase space evolution in ERLs.

Keywords: Energy Recovery, Recirculating Linacs, Instabilities, Free Electron Lasers, Optics 


\section{INTRODUCTION}

There are many ongoing projects which can be used to illustrate the challenges of optics issues for ERLs. Fig. 1 illustrates the range of some of the ongoing projects world wide. It shows how very wide consideration is now being given to the use of the ERL principle to achieve intense, high quality electron beams for a diverse range of applications. In this paper we take examples from each of the categories of projects and discuss the designs and optics issues relevant to those projects. Across each of the designs there are a number of generic optics issues such as designing to minimize disruptive space charge effects, Coherent Synchrotron Radiation (CSR) and beam break-up (BBU) instability etc. Perhaps one of the most important of these issues, given the applications of these accelerators, is the control of the longitudinal dynamics. Only through a deep understanding of the principles and issues in this area can optics designs which will deliver the high quality beams to the various projects be properly conceived. It is this generic topic that we have chosen to review in detail towards the end of this paper.

\section{OPTICS ISSUES FOR THE $4^{\text {th }}$ GENERATION LIGHT SOURCE (4GLS) PROJECT}

\subsection{Introduction to the $4 G L S$ project}

We have used the 4GLS project to illustrate the optic issues being actively considered for this relatively low energy light source during a feasibility design phase. 4GLS is a novel next generation proposal for a UK national light source to be sited at Daresbury Laboratory [1], based on a superconducting energy recovery linac (ERL) with both high average current photon sources (undulators and bending magnets) and high peak current free electron lasers. 
Key features are a high gain, seeded FEL amplifier to generate XUV radiation and the prospect of advanced research arising from unique combinations of sources with femtosecond pulse structure. This suite of sources must cover the whole range from $\mathrm{THz}$ to soft x-ray output and be synchronized for pump-probe and dynamic imaging studies.

The project team is now undertaking design studies and this section outlines some of the optics issues addressed by the initial scoping design work and indicates examples where further studies are required to refine and conclude the conceptual design process. The target is to produce by March 2006 a mature proposal, including a CDR, to support a funding bid.

From its original concept at Daresbury $[2,3,4]$ the linac based source has been considerably developed. Currently the optics design for 4GLS is being studied and evolved with an aim to agree and to finalize major key parameters in the near future in order to allow the projected CDR timescale to be met.

\subsection{Layout of $4 G L S$}

The proposed layout of 4GLS has now progressed from its earlier conceptual level [4] to one that is a realistic scheme that can be simulated and that matches the scientific needs of users. Fig. 2 presents a recent version that contains the essential new features, although arc design has still to be finalized. A $10 \mathrm{MeV}$ superconducting gun injects $\mathrm{CW}$ beam into the 590 $\mathrm{MeV}$ linac and the $600 \mathrm{MeV}$ output beam at $100 \mathrm{~mA}$ traverses the outer path, via various undulator sources and a VUV-FEL, before returning for energy recovery in a second pass. In parallel a beam from a high charge $(1 \mathrm{nC}) \mathrm{RF}$ gun operating at $1 \mathrm{kHz}$ can be accelerated to $160 \mathrm{MeV}$ and then compressed before entering the high energy linac; a third harmonic (3 $\mathrm{GHz}$ ) structure is also inserted at this intermediate energy. The emerging $750 \mathrm{MeV}$ beam is separated from the $600 \mathrm{MeV}$ one by a fixed magnetic chicane and directed through an alternative arc to a further variable energy linac of final output up to $\sim 1 \mathrm{GeV}$. A seeded XUV-FEL is located downstream of this, followed by a long undulator for high energy 
spontaneous radiation. The source portfolio is completed by an IRFEL fed from a separate 50 $\mathrm{MeV}$ linac that is nevertheless synchronized to the high energy ones via its photocathode gun. The team is investigating a modified layout including cascaded injection to possibly reduce the recirculated beam dump power by additional recovery. A preliminary review of the issues related to cascaded injection indicates that whilst the dump challenges could be reduced by chicane injection and the BBU limit potentially raised by reducing the energy ratio of the two beams in a single linac, the disruptive effects of space charge on the quality of the injected beam would have to be assessed in detail to ensure that the required beam quality could be maintained in the cascaded geometry.

At this stage, the baseline design assumes that both the inner, high bunch charge loops and the outer, high average current loops are based on Triple Bend Achromat (TBA) cells. Fig. 3 illustrates the lattice functions through the TBA cell of the 150 degree, 5 cell outer arc for the high average current loop, optimized for isochronous operation (but it can be operated at different values). For this $\mathrm{CW}$ loop the scheme assumes that control of the longitudinal bunch profile through the undulators can be achieved without the use of a "lumped" compressor. The control of the longitudinal dynamics would be achieved by balancing the linac chirp and the $\mathrm{R}_{56}$ of the TBA cells to progressively develop the bunch profile through the various undulators and ultimately optimizing the profile to ensure efficient lasing in VUV FEL.

For the XUV FEL a detailed assessment of bunch compression schemes has reached similar conclusions to other projects: that a third harmonic RF system is an essential feature and that probably compression should be performed in two stages. The alternative method of $\mathrm{T}_{566}$ adjustment with sextupoles in the arc is less attractive because of the impact on overall optics, but has not yet been abandoned. Present computations suggest that an accelerated bunch of length initially below about 2 ps and $0.02 \%$ uncorrelated energy spread can be 
compressed to well below 100 fs. Checks, using ELEGANT [5] with arc bending fields in the range 0.5-1.0 T reveals CSR energy loss below $0.1 \%$ and no significant CSR microbunching problems up to $1 \mathrm{nC}$ charge levels. For the low charge $\mathrm{CW}$ branch of 4GLS increased pre-compression bunch length and energy spread can be tolerated since postcompression energy spread has less importance than for the XUV-FEL. However an upper limit of 7 ps should not be exceeded to avoid severe beam transport non-linearities.

\subsection{Linac Issues}

It is our intention to accelerate the $\mathrm{CW}$ stream of $80 \mathrm{pC}$ bunches (separated by the RF spacing of $1.3 \mathrm{GHz}$ ) from 10 to $600 \mathrm{MeV}$ and every ms to accelerate a $1 \mathrm{nC}$ bunch for the XUV FEL from $160 \mathrm{MeV}$ to $750 \mathrm{MeV}$. A simple sketch of the bunch pattern is given in Fig.4 As well as the usual ERL optics issue of optimizing the lattice to allow the stable transport of two counter propagating beams of the different energy, our present concept assumes that the two beams of different charge can co-propagate through the linac without disrupting the high quality demanded of either beam. Future work is required to determine how this mode can be operated and studies to address a number of issues are currently being initiated, these are listed below.

- $\quad$ BBU, HOM \& Wakefield effects

- Effect on voltage waveform

- Output energy variation of bunches near to the $1 \mathrm{nC}$ bunch

- Phase variation affecting timing and synchronization

- Feedback and feed-forward schemes to minimize disruption

- Tolerance studies

\subsection{Summary and Future Work}

To summarize the novel features of the 4GLS design aimed at providing a unique combinations of sources with femtosecond pulse structure presents the additional optics 
challenge of transporting and accelerating a high quality $\mathrm{CW}$ beam of high average current simultaneously with a $\sim 1 \mathrm{kHz}$, high peak current train of low emittance bunches capable of driving an XUV FEL. Some of the issues involved in this aspect of the design have been identified and will be studied in detail over the coming months. The preliminary arc designs require further optimization and both CSR and space charge effects will be studied in detail as part of full Start-to-End simulations which will include the modeling of the FELs within the design. The studies of these issues will allow the baseline layout to be developed into a feasible, conceptual design for the facility by Early 2006.

\section{OPTICS ISSUES FOR THE ENERGY RECOVERY LINAC PROTOTYPE (ERLP) AT DARESBURY LABORATORY}

\subsection{Introduction}

The second project covered in this paper is the Energy Recovery Linac Prototype (ERLP), currently being built at Daresbury Laboratory. This is an accelerator that will serve as a research and development facility for the study of beam dynamics issues and accelerator technology important to the design and construction of the 4GLS facility. Two major objectives of the ERLP are the demonstration of energy recovery and of energy recovery from a beam disrupted by an FEL interaction as supplied by an infrared oscillator system.

\subsection{Injector}

The injector consists of a high-average current DC photocathode gun, a booster and a transfer line to the main linac. The DC photocathode gun is a replica of the $500 \mathrm{kV}$ Jefferson Lab gun [6] and will operate at a nominal accelerating voltage of $350 \mathrm{kV}$ and bunch charge of $80 \mathrm{pC}$. Electrons will be generated at a GaAs photocathode by the frequency-doubled light $(532 \mathrm{~nm})$ of a mode-locked Nd: $\mathrm{YVO}_{4}$ laser with an oscillator frequency of 81.25 MHz. Two 
solenoids are used for transverse focusing and emittance compensation, and a normalconducting single-cell buncher cavity is utilized to decrease the bunch length from the GaAs cathode. The buncher cavity will be operated at $1.3 \mathrm{GHz}$ and is based on the design employed at the ELBE facility. Electrons are accelerated to an energy of $8.35 \mathrm{MeV}$ in the booster, which consists of two super-conducting 9-cell TESLA-type cavities operated at 1.3 $\mathrm{GHz}$; the cryomodule design is based on the design of the ELBE linac [7]. The layout of the ERLP injector is shown in Fig.5 and a description of the design can be found in [8].

\subsection{Space Charge in the Injector Line}

A full GPT [9] simulation of the injector line including dipoles and space charge is shown in Fig. 6, a comparison with ASTRA [10] is of course not possible as it cannot model dipoles at present. However, we may compare the results given by GPT together with those using the Vinokurov approximation $[11,12]$, and this is shown in the Fig.6 Spikes in the emittance within the dipole magnets are artifacts arising from the way GPT calculates emittances in a curving trajectory: these should be disregarded. There is a remarkably good agreement between the emittance growth estimated analytically and the one modeled in GPT, except at the exit of the last dipole. The reason for the disparity in the last dipole is not yet understood.

\subsection{Beam Transport System}

Electrons from the injector are accelerated to $35 \mathrm{MeV}$ in the super-conducting main linac, which is identical to the booster and composed of two 9-cell TESLA-type cavities. Two $180^{\circ}$ triple-bend achromat (TBA) arcs are used to recirculate the beam to the main linac where the electrons are decelerated to their injection energy and subsequently dumped. A 4dipole chicane provides bunch compression upstream of the wiggler and bypasses the upstream FEL mirror, see Fig. 7. 
The minimum bunch length is required within the wiggler. The compression chicane has a static $\mathrm{R}^{\mathrm{C}}{ }_{56}$ of $0.28 \mathrm{~m}$, which requires an off-crest phase of about $\varphi_{\mathrm{rf}} \approx 9^{\circ}$ in the main linac for full compression. The TBA arcs are able to provide a variable large negative $\mathrm{R}_{56}$. In the nominal setup the first arc is set to $\mathrm{R}^{\mathrm{A} 1}{ }_{56}=0$ whilst the second is tuned to $\mathrm{R}^{\mathrm{A} 2}{ }_{56}=-\mathrm{R}_{56}^{\mathrm{C}}$ to decompress the bunch. The sextupoles in the first arc can be used to linearize the lowestorder curvature induced by the sinusoidal RF during acceleration, by varying $\mathrm{T}_{566}$. The sextupoles in the second arc may be used to minimize the energy spread after deceleration for optimal energy recovery and extraction to the beam dump. Preliminary calculations of resistive-wall and bellows wakefield effects indicate that the induced energy spread from these impedances is small. The $\beta$ functions for the entire lattice of the ERLP are shown in Fig. 8, together with a close-up of the waist through the wiggler in Fig. 9, and the behavior of dispersion in Fig. 10 below.

\subsection{FEL}

The wiggler has been supplied on loan from Jefferson Laboratory, and is a planar device with 40 periods of length $27 \mathrm{~mm}$. The magnet arrays are vertically aligned, giving focusing in the horizontal plane. The matched beam conditions in transverse phase space are thus a waist in the horizontal plane at the wiggler entrance and a waist in the vertical plane at the wiggler centre. This corresponds to a desired $\beta_{\mathrm{x}}$ at the wiggler entrance of $0.5 \mathrm{~m}$, and $\alpha_{\mathrm{y}}=$ 1.75 and $\beta_{\mathrm{y}}=1.25 \mathrm{~m}$ in the vertical plane to give the minimal vertical beam radius averaged along the wiggler.

The optical cavity length is $\mathrm{D}=9.224 \mathrm{~m}$ with the wiggler positioned at the cavity centre; the mirror radii of curvature $\mathrm{R}_{1}$ and $\mathrm{R}_{2}$ are chosen to give a near-concentric cavity with an optical waist at the wiggler centre. The Rayleigh length is $0.75 \mathrm{~m}$ compared to a wiggler length of $1.08 \mathrm{~m}$ - the optimum Rayleigh length for FEL coupling would be less than 
this but would drive the cavity towards instability; the cavity stability is given by $\mathrm{g}_{1} \cdot \mathrm{g}_{2}=$ 0.9 , with $\mathrm{g}_{1}=1-\mathrm{D} / \mathrm{R}_{1}$ and $\mathrm{g}_{2}=1-\mathrm{D} / \mathrm{R}_{2}$.

\subsection{Start-to-End (S2E) Simulations}

The beam dynamics in the high-brightness injector has been modeled including space charge effects using the two codes GPT and ASTRA as described in Section 3.3. The full S2E simulations used the ASTRA code. After the booster, around the arcs the particles have been tracked with the code ELEGANT [5]. The output was examined for any signs of damaging CSR effects but as expected for ERLP this is not a significant issue. The 3D code GENESIS 1.3 was used to model the FEL interaction with the electron beam at $35 \mathrm{MeV}$.

The performance of a free-electron laser (FEL) depends crucially on the electron beam parameters. While analytical calculations can give an estimate of the expected performance, the numerical start-to-end S2E simulations were required to account for various aspects of beam dynamics during the generation, transport and compression of the beam. These studies allowed realistic estimates to be made of the likely large energy spread induced by the FEL process. This was crucial to determine the specification of suitable optics which would allow this disrupted beam to be recirculated for deceleration and then transported into the beam dump [13].

\subsection{Outlook}

The ERLP design has with a relatively straight forward optics design provided a flexible optics which will give the Daresbury physicist with a valuable platform to study the range of beam dynamics issues which are of high importance to optimization of the design for the more complex 4GLS project. These studies will take place during the commissioning and optimization period in late 2006 and 2007. 


\section{THE CORNELL X-RAY ERL PROJECT}

\subsection{Introduction}

This section presents the final light source project overviewed in this paper, and illustrates how the optics issues have influenced the design of an advanced X-ray source based on a relatively high energy ERL. Cornell University has proposed an ERL based synchrotronlight facility which can provide greatly improved X-ray beams due to the high electron-beam quality that is available from a linac. To provide beam currents competitive with ring-based light sources, the linac must operate with energy recovery, the feasibility of which we plan to demonstrate in a downscaled prototype ERL. Here we present two of several $5 \mathrm{GeV}$ ERL upgrade possibilities for the existing 2nd generation light source CHESS at CESR. This proposed upgrade suggests how existing storage rings can be extended to ERL light sources with much improved beam qualities. Since today's ring-based light sources have beam energies of several $\mathrm{GeV}$ and beam currents a sizable fraction of an Ampere, Cornell is planning a facility that can deliver $5 \mathrm{GeV}$ beams of $100 \mathrm{~mA}$. Continuous beams of these currents and energies would require klystrons delivering a power of the order of a GW to the beam. Without recovering this energy after the beam has been used, such a linac is impractical.

DC photo-emission sources with negative electron affinity cathodes have been simulated to give less than $0.4 \pi \mathrm{mm}$ mrad for a $100 \mathrm{~mA}$ beam current in a continuous beam at 1.3 GHz. [14]. However, the large beam powers and small transverse and longitudinal emittances required for an X-ray ERL have not been achieved anywhere. CESR has been used

for the high-energy physics experiment CLEO and as the $5 \mathrm{GeV}$ second generation light source CHESS since its construction and it will be available for X-ray physics alone when CESR stops high energy physics operation. Then we plan to upgrade CHESS to an ERL facility based on the CESR complex. 
Cornell University is currently prototyping a DC photoemission electron source and a $10 \mathrm{MeV}$ injector linac [15] for low emittance beams of high CW currents [16]. The bunches that this injector is designed to produce [17] could be accelerated in the planned X-ray ERL. An X-ray ERL will enlarge the wide range of applications of third generation light sources by producing beams similar to their $\mathrm{CW}$ beams, albeit with much higher brilliance due to the much smaller horizontal emittance and possibly smaller energy spread. At the same time, it can serve more specialized experiments that require ultra small emittances for high spatial resolution or ultra short bunches for high temporal resolution [18]. Three different operation modes are planned, one for high flux, one for high brilliance, and one for short bunches. Parameters for these operating modes, not containing the smallest simulated emittances, are shown in Table. 1.

The design of the Cornell X-ray ERL should be made cost efficient by reusing much of CESR's infrastructure. The operation of CHESS should be disrupted as little as possible while building and commissioning the ERL, the facility should provide space for a sufficient number of X-ray beamlines. While it could have turned out that reusing CESR imposes too many constraints, quite contrary it has been found that the flexibility of CESR's magnet arrangement holds several advantages for an ERL design. First and second order electron optics have been found for bunch compression down to at least $100 \mathrm{fs}$, and nearly all required magnet strength could be supported by the magnets that are in CESR today. In order to extend the space for cavities, to make space for possible upgrades, and to minimize the impact on CHESS operation, work has been invested in the layout of Fig. 11. It shows the CESR tunnel and the layout of a possible linear ERL extension. Electrons from a $10 \mathrm{MeV}$ injector (1) would be accelerated to the right in a $2.5 \mathrm{GeV}$ linac (2). A return loop (3) would send them into a second linac which is located in the same straight tunnel (4) and accelerates to $5 \mathrm{GeV}$. An arc (5) injects the electrons into the CESR ring (6) where they travel counterclockwise until another 
arc (7) injects them back into the first linac, where they are decelerated to $2.5 \mathrm{GeV}$. The return loop leads the electrons to the second linac section where deceleration back to $10 \mathrm{MeV}$ and leads to the beam dump (8). The South half of the CESR tunnel would contain undulators and would reuse the current facilities of CHESS. Additionally, new user areas could be created in the North section of CESR (at the top of the figure) and in straight sections of the linac tunnel. The location of the linac at a hillside is chosen in such a way that no existing building foundations interfere and that X-ray beamlines with easy access can be added between the linac and CESR.

A return arc is also shown which connects the arcs (5) and (7) so that electrons can return to the linacs after acceleration without passing through CESR. This connection has been chosen so that the ERL could be built and commissioned while CESR is still used as a storage ring light source. Other advantages of this upgrade plan are that all of the CESR tunnel is reused, which creates space for a large number of insertion devices.

To limit the cost of cooling, the accelerating gradient of the SC cavities should not exceed $20 \mathrm{MV} / \mathrm{m}$. Thus, $250 \mathrm{~m}$ of cavities would lead to $5 \mathrm{GeV}$ beam energy. However, much more space is required for the linac, since higher order mode (HOM) dampers and connecting tubes have to be placed after each cavity and 2 quadrupoles have to be placed after each cryomodule of ten 7-cell cavities. Our analysis, which is based on the $1.3 \mathrm{GHz}$ cavity cell shape of the TESLA design, on four HOM couplers of the TTF type per cavity, and on one ferrite HOM damper of the CESR type per cavity, showed that for a beam tube radius of 39 mm we could not obtain a fill factor larger than 53\%. The total linac length would therefore have to be about $500 \mathrm{~m}$. The tunnel extension shown in Fig. 11 has a section of $250 \mathrm{~m}$ with two linacs side by side. A sketch of a possible tunnel cross-section is shown in Fig. 12. A straight tunnel housing two linacs, reduces tunnel cost as well as the required length of cryogenic lines 
and cables. The tunnel is laid out longer than required for the two linacs, so that an extension of the facility by extra undulators or by an FEL is possible.

\subsection{Arc optics}

We studied whether a favorable optics can be found for the CESR South arc in spite of the constraints imposed by the existing tunnel. To reuse as much as possible from CESR, we maintained the bending magnets and quadrupoles in their current positions and only replaced the regions where the undulators would be installed. Each of 7 undulator has two matching quadrupoles at each side and is separated from the next undulator by a three-bend achromate. Finding an optics for the operation with $2 \mathrm{ps}$ bunch length turned out to be relatively simple. The matching constraint were $\beta=1 \mathrm{~m}, 2.5 \mathrm{~m}, 2.5 \mathrm{~m}, 12.5 \mathrm{~m}, 2.5 \mathrm{~m}, 2.5 \mathrm{~m}, 1 \mathrm{~m}$ in the seven successive undulators, and $\alpha=0, \mathrm{D}=0, \mathrm{D}^{\prime}=0$ in these seven places. The optics for an rms bunch length of $100 \mathrm{fs}$ has to ful-fill several additional requirements. The RF acceleration phase $\varphi$ and the first and second order time of flight terms $\mathrm{R}_{56}$ and $\mathrm{T}_{566}$ of the first half of the arc have to be chosen to yield the desired bunch length in the central undulator [19]. For the second half of the return arc, $\mathrm{R}_{56}$ and $\mathrm{T}_{566}$ are determined by minimizing the energy spread after deceleration. The beta function and the dispersion for the return arc are shown in Fig. 13. Even though the magnet arrangement is symmetric around the center of the arc, the optics functions are not symmetric since the conditions for $\mathrm{R}_{56}$ and $\mathrm{T}_{566}$ are different for the two halves. The second order time of flight term $\mathrm{T}_{566}$ is influenced by sextupoles and has to have the same sign as $\mathrm{R}_{56}$. This is hard to achieve in the achromatic arrangements that have been proposed for this purpose. However, with the FODO like optics of the CESR arc this can be achieved with relatively weak sextupoles. This advantage is due to the large dispersion after the linac. The nonlinear dynamics in sextupoles can increase the emittance. However, due to the weak sextupoles and the small transverse beam size, the dynamics is so weakly nonlinear that only the second order dispersion $\mathrm{T}_{166}$ and its slope had to be eliminated in the center of the return 
arc. The second order conditions on $\mathrm{T}_{566}, \mathrm{~T}_{166}$ and $\mathrm{T}_{266}$ were satisfied by three sextupoles on each side of the arc close to the three maxima of the dispersion in Fig. 13. For short-bunch operation, coherent synchrotron radiation (CSR) can also increase the emittance. The emittance growth was computed with the code ELEGANT and is shown in Fig. 14. Since the beam dilution due to the nonlinear dispersion is included, the emittance is shown to decrease where the second order dispersion is corrected. In the central undulator, the emittance for $100 \mathrm{fs}$ bunch length has only increased by a factor of 1.8 . To limit the emittance growth, it was found prohibitive to compress the bunch length to its minimum since this creates a spike in the longitudinal density and strongly enhances CSR.We therefore increased $\varphi$ to obtain $100 \mathrm{fs}$ bunches without full compression.

To the 7 undulators in the South section and the 7 in the North section of CESR, additional undulators could be placed in the section between the linac and CESR, which has been designed with a gentle arc of achromats.

\section{Two linacs and return loop}

The loop (3) connecting the two linacs was chosen so as to produce an acceptable emittance increase due to synchrotron radiation. Fig. 15 shows an optics with 16 achromatic cells. The magnet in the center of each cell has a negative bend to make the lattice isochronous, it has a horizontally focusing quadrupole which produces a very small average horizontal beta function, and it has a sextupole to correct the second order dispersion. After this correction, nonlinear dynamics does not lead to emittance growth for a $0.2 \%$ energy spread beam that one obtains for $6^{\circ}$ off-crest acceleration, as required for compressing a 2 ps long bunch to $100 \mathrm{fs}$ after the linac. This loop could also be used for energy spread reduction by running the second linac $-6^{\circ}$ off-crest as discussed in [19].

The emittance growth for a $100 \mathrm{~mA}$ beam due to incoherent synchrotron radiation for the high flux option in Table. 1 is $0.04 \mathrm{~nm}$ and therefore acceptable. The emittance growth due 
to coherent synchrotron radiation as computed by ELEGANT [6] is shown in Fig. 16. The fluctuations are due to second order dispersion, but the difference between the two curves shows the influence of coherent synchrotron radiation. It is approximately $0.006 \mathrm{~nm}$ and thus negligible.

The optics for the linear accelerator is shown in Fig. 17 in $x$ and $y$ for the accelerating beam. Both linacs are shown, but the optics of the return loop is not shown in between them. The optics for the accelerating beam is shown, that for the decelerating beam of the ERL is mirror symmetric. The beta functions are relatively small. The threshold current of the beam breakup (BBU) instability has been calculated for a similar optics [20]. For quite pessimistic assumptions (HOMs with R/Q of $100 \Omega$ and $\mathrm{Q}=10^{4}$ ) the threshold current is about $200 \mathrm{~mA}$ for a HOM frequency randomization of $1.3 \mathrm{MHz}$. When the modes are polarized and an optics is chosen that couples horizontal oscillations to the vertical and vice versa [21], the threshold current is about $650 \mathrm{~mA}$.

Emittance growth due to coherent synchrotron radiation is a phenomenon which is hard to compute accurately. We are therefore also investigating alternate designs which minimize the total bend angle of the ERL similar to what was presented in [22]. A possible layout that is adjusted to the geography of the Cornell campus is shown in Fig. 18.

\subsection{Beamlines}

The South half of the CESR tunnel would contain undulators and would reuse the current facilities of CHESS. Additionally, new user areas could be created in the North section of CESR (at the top of Fig. 11) and in straight sections of the linac tunnel. The location of the linac at a hillside is chosen in such a way that no existing building foundations interfere and that X-ray beamlines with easy access can be added between the linac and CESR. The outline and the optics for this section is shown in Figs. 19 and 20. The presented optics of Fig. 13 modifies CESR as little as possible. It contains four undulators of $5 \mathrm{~m}$ 
length, two undulators of $2 \mathrm{~m}$ length and one $25 \mathrm{~m}$ long undulator in the South, and an equivalent arrangement could be added in the North. The beta functions are $1 \mathrm{~m}, 2.5 \mathrm{~m}$ and $12.5 \mathrm{~m}$ in the center of these undulators respectively but a flexible lattice can produce larger beta functions easily. Currently 18 beamlines and their science case are being investigated. The undulator lengths are: $25 \mathrm{~m}$ for $2,5 \mathrm{~m}$ for $9,2 \mathrm{~m}$ for $2,1 \mathrm{~m}$ for $1,2 \mathrm{~m}$ for 3 undulators in the $2.5 \mathrm{GeV}$ loop in the East, and one diagnostic undulator. These studies cover the areas of: phase imaging and topography, coherent diffraction and XPCS microscopy on the nm scale, nanoscope and nanoprobe TXM and STXM to nm resolution, protein crystalography, inelastic X-ray scattering, femtosecond timing, resonant scattering, SAX and XPCS for mesoscopic science, and general material science, e.g. at high pressures. The undulators at $2.5 \mathrm{GeV}$ would be for soft X-ray studies.

\subsection{Optics}

The loop (3) connecting the two linacs was chosen so as to produce an acceptable emittance increase due to synchrotron radiation. After appropriate nonlinear correction, the dynamics does not lead to emittance growth for a $0.2 \%$ energy spread beam that one obtains for $6^{\circ}$ off-crest acceleration, as required for compressing a 2 ps long bunch to $100 \mathrm{fs}$ after the linac. This loop could also be used for energy spread reduction by running the second linac $-6^{\circ}$ off-crest as discussed in [19].

\subsection{Summary}

By addressing the major optic issues at the design stage, it has been shown that an extremely advanced light source can be designed on the Cornell site which would provide a very high quality and versatile range of X-Ray sources from a number of undulators. 


\section{OPTICS ISSUES FOR ERL PROJECTS AT BNL}

\subsection{Introdution}

Looking at the proposed energy recovery linac(ERL) based accelerators at the Brookhaven National Laboratory provides an opportunity to consider ERL optics issues from the perspectives of designer whose primary aims is to produce beams satisfying the challenging demands of the high energy and nuclear physics communities. The Collider-Accelerator Department (C-AD) at Brookhaven National Laboratory is pursuing development of ERL-based accelerators for multiple applications in high energy and nuclear physics [23]. These programs are aimed towards a ten-fold luminosity increase at the Relativistic Heavy Ion Collider (RHIC) [24] and development of an ERL-based electron-ion collider, known as eRHIC [25,26]. As part of this program, we are designing, constructing and commissioning a dedicated ERL for R\&D studies of issues relevant to operating with high current, high brightness electron beams [27]. All three ERLs at BNL will be based on a super-conducting RF cavities (guns, linacs, etc.) operating at a frequency of $703.75 \mathrm{MHz}[23]$.

\subsection{Optics issues of $B N L$ 's $R \& D E R L$}

The R\&D ERL facility at BNL, shown schematically in Fig. 21, aims to demonstrate CW operation of an ERL with average beam current in the range of 0.1 - 1 ampere, combined with very high efficiency of energy recovery. Projected parameters of this ERL are summarized in Table 2. Electrons are generated and accelerated in a superconducting halfcell gun to about 2.5-3.5 MeV. They are injected into the ERL loop through a zigzag merging system, a novel dispersion-free merger for space charge dominated beams incorporating an emittance compensation scheme (see Ref. [28] in this issue). Two solenoids are used for transverse focusing in the injection system. An SRF 5-cell linac [23,29] accelerates electrons up to $20 \mathrm{MeV}$, and the accelerated electron beam passes through a return loop comprised of two achromatic arcs with a straight section between them before 
returning to the same linac. The path-length of the loop provides for $180^{\circ}$ change of the RF phase, causing electron deceleration in the linac (hence the energy recovery) down to 2.5 $\mathrm{MeV}$. The decelerated beam is separated from the higher energy beam and is directed to the beam-dump.

The flexible lattice of the ERL, shown in Fig. 22 has 29 individually controlled quadrupoles and provides for independent control of the most important elements of the transport matrix:

$$
\left[\begin{array}{c}
x \\
x^{\prime} \\
y \\
y^{\prime} \\
-c \delta t \\
\delta E / E
\end{array}\right]_{s 2}=\left[\begin{array}{cccccc}
R_{11} & \boldsymbol{R}_{12} & \ldots & \ldots & \ldots & D_{x} \\
R_{21} & R_{22} & \ldots & \ldots & \ldots & D_{x}{ }^{\prime} \\
\ldots & \ldots & R_{33} & \boldsymbol{R}_{34} & \ldots & D_{y} \\
\cdots & \ldots & R_{43} & R_{44} & \ldots & D_{y}{ }^{\prime} \\
\ldots & \ldots & \ldots & \ldots & R_{55} & \boldsymbol{R}_{56} \\
\cdots & \ldots & \ldots & \ldots & \ldots & R_{66}
\end{array}\right] \cdot\left[\begin{array}{c}
x \\
x^{\prime} \\
y \\
y^{\prime} \\
-c \delta t \\
\delta E / E
\end{array}\right]_{s 1}
$$

The adjustable part of the lattice has two arcs and a straight section. Each arc consists of the two 60-degree chevron dipoles, two 30-degree dipoles with parallel edges, and three quadrupole triplets. A bending radius of $20 \mathrm{~cm}$ was chosen for the dipoles to provide a possibility of using the visible part of synchrotron radiation spectrum from a $20 \mathrm{MeV}$ electron beam for beam diagnostics. The chevron 60-degree dipoles split the focusing between vertical and horizontal directions in the dipoles. The quadrupole triplets between the dipoles allow us to control the value and the sign of longitudinal dispersion, while keeping the arcs achromatic. Eight quadrupoles in the dispersion-free straight section provide for matching of the $\beta$-function and for choosing the desirable phase advances independently in the horizontal and vertical planes. The lattice has complete independent control of these values and the signs of matrix elements $R_{12}$ and $R_{34}$, which are critical for transverse stability, and $\mathrm{R}_{56}$, which is critical for longitudinal stability of the beam. The optics functions for one turn for the case of $\mathrm{R}_{56}=0$ is shown in Fig. 23. The loop has a full assortment of 
horizontal and vertical correctors and beam position monitors. The ERL has been under construction since 2004 and commissioning is planned in 2007. This ERL will serve as a testbed for testing the limits of transverse and longitudinal stabilities of intense ampere-class CW electron beams.

Space charge effects play a very significant role in the R\&D ERL and require careful start-to-end simulations. Beam-size envelopes for $1.4 \mathrm{nC}$ and $10 \mathrm{nC}$ electron bunches starting from the photocathode to the exit from the ERL were obtained with PARMELA [30], and are shown in Fig. 24. The remaining R\&D ERL beam parameters are summarized in Table 2.

\subsection{Optics issues of e-Cooler ERL}

The RHIC electron cooler, currently in an R\&D phase, requires a $54 \mathrm{MeV}$ electron beam, which cannot be produced by an electrostatic accelerator. R\&D of electron cooling in RHIC [31] leads to the following electron beam parameters: energy of $54 \mathrm{MeV}$, bunch charge of 20 $\mathrm{nC}$, bunch repetition frequency of $9.4 \mathrm{MHz}$, energy spread of the order of $10^{-4}$, RMS normalized emittance better than 40 microns, magnetization of about 2 to 5 Tesla $\mathrm{mm}^{2}$. The parameters of an electron beam required for electron cooling of RHIC are similar to those of the R\&D ERL, but the ERL for these systems have some significant differences. The ecooler ERL [32], shown in Fig. 25, will operate with a magnetized electron beam generated from a cathode submerged in a solenoidal magnetic field. When electrons leave the solenoidal field, the magnetization is transferred into mechanical angular momentum equivalent to a very large correlated emittance when projected onto $x-x^{\prime}$ and $y-y$ ' phasespace planes. This magnetization, i.e. the correlations, must be preserved through the entire acceleration and transport process to be used in the cooling solenoid. This requirement significantly complicates the ERL lattice. 
One present design, shown in Fig. 25, uses flat-to-magnetized beam converters [33] to preserve the quality of the electron beam. A number of cavities operating at the fundamental (703.75 MHz), $3^{\text {rd }}$ harmonic $(2.1 \mathrm{GHz})$ and sub-harmonic $(200 \mathrm{MHz})$ are used to provide matched length and low energy spread of the electron beam in the cooling section. Two achromatic stretcher loops with a large value of $\mathrm{R}_{56}$ (and two $200 \mathrm{MHz}$ RF cavities) are used to initially increase the bunch length of the electron bunch from a few millimeters to about 30 $\mathrm{cm}$ (i.e. the length of ion bunches in RHIC) and to compress it back after it passed the cooling section.

We must also decouple the cooling section for the hadron beam. To accomplish this, the cooling solenoid is split into two equal sections, with opposite field directions, giving a zero net hadron beam coupling. This requires a change in the direction of the electron beam magnetization between the two opposite solenoid sections, which will be achieved [34] using a quadrupole matching section providing a phase advance of 180 degrees and a vertical phase advance of 360 degrees. Fig. 26 shows the optics for this matching section.

\subsection{Optics issues of ERL-based eRHIC}

The detailed beam parameters and proposed configurations for an ERL-based polarized electron-hadron collider eRHIC are presented in Refs. [23,25]. Table 3 gives a brief summary of the electron beam parameters for the collider and light source mode of eRHIC operation. Two of the most attractive features of ERL based eRHIC are full spin transparency of the ERL at all operational energies and the capability to support up to four interaction points. Issues related to spin transparency are described in $[23,25]$.

The parameters of the polarized electron beam are very impressive and this facility could easily be used as a next generation light source. In addition, RHIC rings operate for only about 30 weeks per year, which leaves about 4 and a half months for dedicated mode of ERL 
operation as a light source. In this case unpolarized electrons with lower charge per bunch and a higher rep-rate will be used, while keeping the same level of average electron beam current $(\sim 500 \mathrm{~mA})$. These parameters of the electron beam in eRHIC's ERL naturally match the requirements for a next generation light source.

The ERL for eRHIC operates on the same principles as the R\&D ERL, with some differences: the eRHIC electron gun will generate longitudinally polarized electrons using GaAs photocathode driven by a high power circularly polarized laser beam from a small size free-electron laser (also driven by a smaller ERL) [25].

There are two designs of ERL for eRHIC: a 5-10 GeV stand-alone ERL (see Fig. 27) and a 5$20 \mathrm{GeV}$ ERL with the return loops located in the RHIC tunnel (see Fig. 5 in Ref. [23]). Both designs have low and high energy loops to separate the function of generating and disposing the electron beam from the main acceleration process. Furthermore, the high energy of electrons in eRHIC causes a significant loss of energy at the MeV scale, which must be compensated by a special RF system.

The stand-alone ERL has four major and two minor arcs, as shown in Fig. 28. It is comprised of a rather simple FODO lattice with high density, but low field dipoles for reducing synchrotron radiation losses to about $5 \mathrm{MeV}$ for the entire cycle from the gun to the dump. The arc with the largest energy of electrons incorporates the interaction point (IP). Polarized electrons with initial energy of $5 \mathrm{MeV}$ are injected into the first ERL with a 500-MeV superconducting linac. They pass twice through this linac before entering the main ERL. The main ERL has two linacs with a nominal energy gain of $2250 \mathrm{MeV}$ per linac. Passing twice through each linac, the electron beam reaches a maximum energy of $10 \mathrm{GeV}$. During the process of acceleration in the ERL, the electron beam passes through the arcs where it loses about $2.5 \mathrm{MeV}$ of its energy in the form of synchrotron radiation. At full energy, the electron beam passes through a half arc towards the IP. A Compton laser polarimeter installed in the 
dogleg just before this acquires final measurements of the electron beam's polarization in the IP. Each normal arc or transfer line of the ERL ensures a delay in electron beam time equal to an integer number of RF cycles. In this case, all linacs will synchronously accelerate (or decelerate) electrons. The last arc with the IP and vertical chicane is a special one - it provides for a delay in the electron beam time equal to an integer number plus a half of RF cycles to change the accelerating sequence into a decelerating one. It also incorporates a special cavity to compensate for the synchrotron radiation loss.

The main ERL-based design of eRHIC is based on the main arcs sharing the $3.8 \mathrm{~km}$ circumference RHIC tunnel, Fig. 29. Leveraging the RHIC tunnel for ERL's arcs significantly increases the radii of curvature and reduces synchrotron radiation loss. With $85 \%$ filling factor for the arcs, the $10 \mathrm{GeV}$ ERL will have energy loss of less than $2 \mathrm{MeV}$ per pass and very low linear power density of synchrotron radiation of $0.22 \mathrm{~kW} /$ meter. The linear power density of synchrotron radiation will reach the level of $8.8 \mathrm{~kW}$ per meter, presently attained at the B-factory, for a $25 \mathrm{GeV}$ ERL.

On the way to the final energy the electron beam passes through the arcs, where synchrotron radiation may significantly affect the electron beam parameters. Our present design is based on 150 25-meter long achromatic cells (see Fig. 3 in Ref. [27]) with a bending radius of 400 $\mathrm{m}$ in the dipoles. The total synchrotron radiation energy loss is $35 \mathrm{MeV}$ for $20 \mathrm{GeV}$ electrons. This rather mundane triplet lattice in the main arcs [27] provides for complete preservation of the transverse emittance (to be exact, the horizontal emittance grows for $1.610^{-3} \mathrm{~nm}-\mathrm{mrad}$ for a $20 \mathrm{GeV}$ beam). At the same time, the quantum fluctuations of synchrotron radiation determine the relative energy spreads for both $10 \mathrm{GeV}$ and $20 \mathrm{GeV}$ electron beams, which still remain very low at 2.5-to-5 $10^{-5}$ level [27].

Using the ERL with a low emittance electron beam dramatically simplifies the lattice of the interaction region $[25,35]$. Single pass interaction of electrons with the ions and very low 
emittance of the electron beam allows the use of large $\beta^{*}(\sim 1$ meter $)$ for the electron beam, while matching the transverse size of electron and hadron beams. Smaller electron beam emittance results in a 10-fold smaller aperture and angular acceptance requirements for the electron beam and permits movement of the focusing quadrupoles for the electron beam outside the detector and the IP region, while leaving the dipoles to separate the beams. Therefore, the electron side of the IP optics does not present significant new challenges. Collisions with hadrons significantly modify the distribution of the electron beam (see Fig. 30). This requires tuning of the ERL optics during operation depending on the hadron beam intensity. A triplet following the IP can easily accomplish this. The main challenge of the IP design is the careful evaluation and protection of the detector from direct and scattered synchrotron radiation $[36,37]$.

Overall the ERL part of the eRHIC lattice has many subtle nuances but does not present significant new engineering design challenges. Many aspects of the ERL optics (for example, the transverse and longitudinal stability) in eRHIC will be addressed in the R\&D FEL as well in the e-cooler ERL. The $20 \mathrm{GeV}$ ERL for eRHIC will have the same optics issues, but within a system of much larger scale.

The real challenges of these projects are in the generation of high-current electron beams with a high degree of polarization, the preservation of beam quality in the gun and merging sections, and the damping of the low-energy decelerated electron beam. Many of these issues will be addressed in the R\&D ERL program at C-AD using low-energy electron guns and ERLs.

\section{LONGITUDINAL PHASE SPACE MANIPULATION AT MEDIUM AND HIGH ENERGY: APPLICATION TO ENERGY-RECOVERY LINACS}

\subsection{Introduction}


Finally in this section we address the generic issue of longitudinal phase space evolution in an ERL which requires special attention particularly for ERLs where the quality of beam delivered is critically dependant on this single pass dynamics. To zeroth order, after the beam has been used (e.g. participated in the free-electron laser (FEL) process), it has to be reinjected with the proper decelerating phase. This requires the recirculation loop to be $\sim$ (n + 1/2) $\lambda_{\mathrm{rf}}$ where $\mathrm{n}$ is an integer and $\lambda_{\mathrm{rf}}$ is the wavelength associated to the radio-frequency (rf) accelerating system. To higher order, the longitudinal phase space correlations between an electron relative longitudinal coordinate ${ }^{1} \mathrm{z}$ and its fractional momentum offset $\delta$ needs to be properly chosen such as minimizing the final fractional momentum spread after deceleration. In this Note we first follow the approach presented in Reference [13] and consider the simple case of linear longitudinal manipulation, we then build on this simple treatment and consider the effects of longitudinal space charge (LSC). We also address possible issues pertaining to coherent synchrotron radiation (CSR).

\subsection{Linear longitudinal dynamics}

We first discuss the requirement on the decelerating phase $\varphi^{\prime}$ and consider an electron being accelerated by the accelerating voltage $V_{r f}$ and phase $\varphi$. The electron energy gain is $\Delta E$ $=\mathrm{e} \mathrm{V}_{\mathrm{rf}} \cos \varphi$. Proper energy recovery requires the energy loss of the electron being decelerated to be opposite to the energy gain of the accelerated electron. This requirement alone imposes two values for the decelerating phase $\varphi^{\prime}{ }_{ \pm}=\pi \pm \varphi$.

\footnotetext{
${ }^{1}$ In our convention $z>0$ corresponds to the bunch tail
} 
Let's now consider the requirement on the linear correlation between $\mathrm{z}$ and $\delta$. The energy gain for an electron with longitudinal coordinate $\mathrm{z}_{\mathrm{A}}$ is $\Delta \mathrm{E}_{\mathrm{A}}(\mathrm{s})=\mathrm{eV}_{\mathrm{rf}}\left[\cos \left(\mathrm{kz}_{\mathrm{A}}+\varphi\right)-\cos \varphi\right]$

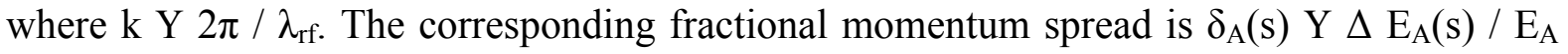
where $\mathrm{E}_{\mathrm{A}}=\mathrm{E}_{0}+\mathrm{e} \mathrm{V}_{\mathrm{rf}} \cos \varphi$. The linear correlation imparted in $\left(\mathrm{z}_{\mathrm{A}}, \delta_{\mathrm{A}}\right)$ is $\left.\mathrm{r}_{\mathrm{A}} \mathrm{Y} \frac{d \delta_{A}}{d z_{A}}\right|_{z_{A}=0}=-\frac{e V_{r f}}{\varepsilon_{A}} \sin \varphi$. After acceleration the beam propagates through a recirculation loop characterized by its overall momentum compaction $\mathrm{R}_{56}$. When no source of energy spread is taken into account, the longitudinal motion due to the non-isochronicity of the loop may alter the longitudinal phase space correlation. The correlation coefficient downstream of the recirculation loop is thus given by $r_{D^{-}}=\frac{r_{A}}{1+r_{A} R_{56}}$. Similarly to the acceleration, the deceleration induces a linear correlation given by $r_{D}=\frac{e V_{r f}}{\sin \varphi^{\prime}}$, where $\mathrm{E}_{\mathrm{D}}$ is the energy after deceleration. The main goal of the overall longitudinal phase space manipulation is to reduce the fractional momentum spread of the decelerated beam. Ideally the cancellation is achieved, to first order, when

$r_{D^{-}} \frac{\varepsilon_{A^{-}}}{\varepsilon_{D}}+r_{A}=0$

Practically it is instructive to consider the two cases associated to the possible decelerating phase values $\varphi^{\prime}{ }_{ \pm}$. Case (1): the recirculation loop is isochronous $R_{56}=0$ and Eq. (1) is verified for $\varphi^{\prime}=\phi+\pi$. Case (2): the recirculation loop is set-up as a reflector $\left(r_{A} R_{56}=-2\right.$ or $\mathrm{Z} \rightarrow-\mathrm{Z}$ ) and Eq. (1) requires $\varphi^{\prime}=\pi-\varphi$. These two cases are illustrated in Fig. 31. Case (2) is generally undesired since it implies the bunch is over-compressed and collective effects such as CSR might be detrimental to the beam quality (see below). 
We now include in the model the effects of longitudinal space charge (LSC) on the correlation coefficient. This is motivated by recent observations at the JLab $10 \mathrm{~kW}$ FEL [38]. The topics has already been addressed in Ref. [39]. Given the on-axis longitudinal electric field associated to LSC [40], we derive the induced linear correlation in the longitudinal phase space

$$
\left.r_{L S C} \mathrm{Y} \frac{d \delta_{S C}}{d z}\right|_{z=0}=\frac{g_{0} \hat{I}}{I_{A} \sigma^{2}{ }_{z} \gamma}\left\{\begin{array}{l}
\mathrm{L} / \gamma^{2} \text { for a drift } \\
\mathrm{L} / \gamma_{0} \gamma \text { in an accelerating section }
\end{array}\right.
$$

The longitudinal charge distribution is assumed to be a Gaussian with rms length $\sigma_{\mathrm{z}}$. $\mathrm{L}$ is the length of the considered section, $\mathrm{g}_{0}$ is a geometric factor ${ }^{2}, \mathrm{I}_{\mathrm{A}}=17 \mathrm{kA}$ is the Alfvèn current and $\hat{\mathrm{I}}=|\mathrm{Q}| \mathrm{c} /\left(\sqrt{2 \pi} \sigma_{\mathrm{z}}\right)$ is the peak current within the bunch $(\mathrm{Q}$ is the bunch charge). The Lorentz factors $\gamma$ and $\gamma_{0}$ correspond respectively to the energy at which Eq. (2) is evaluated and to the initial energy when acceleration is considered ${ }^{3}$. LSC introduces a positive correlation in our convention: the tail (resp. head) of the bunch looses (resp. gains) energy. When the full recirculation is taken into account the total LSC-induced change of correlation is

$$
r_{L S C, \text { tot }} \approx \frac{g_{0} \hat{I}}{I_{A} \gamma_{A}}\left[2 \frac{L_{\text {linac }}}{\sigma_{z, \text { linac }}^{2} \gamma_{0} \gamma_{A}}+\frac{L_{\text {recirc }}}{\sigma_{z, \text { recirc }}^{2} \gamma_{A^{2}}}\right]
$$

${ }^{2} \mathrm{~g}_{0}=1+\frac{1}{2} \log (\mathrm{b} / \mathrm{a})$ for a transverse uniform bunch of radius a propagating in a perfectly conducting pipe of radius $b$

${ }^{3}$ we assume the acceleration is of the form $\gamma(s)=\gamma_{0}+\gamma^{\prime} s$, where $\gamma^{\prime}=d \gamma / d s$ 
where $\sigma_{z,\{\text { linac,recirc }\}}$ are the average bunch length in the accelerating (or decelerating) and recirculation sections, and $\mathrm{L}_{\text {linac,recirc }}$ are the length of the sections. Considering the numerical values experimentally achieved at the JLab $10 \mathrm{~kW}$ FEL [38], $\sigma_{\mathrm{z}} \approx 0.5 \mathrm{~mm}, \mathrm{Q}=135 \mathrm{pC}, \gamma=$ 160 assuming $\mathrm{g}_{0}=3$ and taking a drift length $\mathrm{L}=130 \mathrm{~m}$, we obtain $\mathrm{r}_{\mathrm{LSC}} \approx 2.3 \mathrm{~m}^{-1}$. The latter value translates into a needed change of $\sim 5^{\circ}$ in the accelerating section phase in order to maintain the same correlation as the one that would be achieved without accounting for LSC (we assume $\mathrm{E}_{\mathrm{A}}=80 \mathrm{MeV}$ and $\mathrm{eV}_{\mathrm{rf}}=71 \mathrm{MeV}$ ). Because of the significant impact of LSC, the matching condition Eq. (1) has to be modified by changing $r_{A}$ into $r_{A}+r_{L S C \text {,tot }}$.

Practically the recirculation transport also provides local manipulation of the longitudinal phase space. For instance a local dispersion bump conveniently located compresses the bunch. In this case the upstream accelerating linac has to be operated off-crest. Introducing the magnetic compressor momentum compaction $\mathrm{R}_{56, \mathrm{BC}}$, the rms bunch length after compression is related to the initial bunch length $\sigma_{z_{0}}$ and relative fractional momentum spread $\sigma_{\delta_{0}}$ via

$\sigma_{z, B C}=\left[\mu^{2} \sigma_{z_{0}}^{2}+\left(R_{56, B C} \sigma_{\delta_{0}} \frac{\varepsilon_{0}}{\varepsilon_{A}}\right)^{2}\right]$

where $\mu$ Y $1+r_{A} R_{56, B C}$. The remaining longitudinal lattice downstream of the bunch compressor area (e.g. after a wiggler) is set-up to provide a momentum compaction opposite to the bunch compressor $\left(\mathrm{R}_{56, \mathrm{R}}=-\mathrm{R}_{56, \mathrm{BC}}\right)$ thereby vanishing the total momentum compaction of the recirculation loop ( since $\mathrm{R}_{56}=\mathrm{R}_{56, \mathrm{R}}+\mathrm{R}_{56, \mathrm{BC}}$ ). Because the minimum achievable bunch length is $R_{56, B C} \sigma_{\delta_{0}} \frac{\varepsilon_{0}}{\varepsilon_{A}}$, compressing the bunch to its maximum should be avoided, especially at high energy, due to possible detrimental collective effects. 
In the JLab IR-Demo, the bunch was going through three longitudinal waists during the recirculation [41], and despite the relatively low energy $(\mathrm{E}=40 \mathrm{MeV})$ and modest uncorrelated energy spread $\left(\mathrm{E} \sigma_{\delta_{0}} \approx 5 \mathrm{keV}\right)$, significant energy modulation at $60 \mathrm{pC}$ was observed; see Fig. 32.

\subsection{Nonlinear effects and cures}

The linear approach in the previous Section breaks as soon as sources of momentum spread dilutions, e.g. induced by the FEL process, are included. The resulting large fractional momentum spread (and thus the longer bunch after deceleration) requires the previous treatment to include second and possibly higher order terms in z. Nonlinear effects should indeed be included starting from upstream of the injector especially since in common present injector designs the bunch is kept long in order to mitigate transverse emittance growth due to space charge. Then, during acceleration in the linac, the longitudinal phase space accumulates nonlinear correlations introduced by the cosine-like dependence of the accelerating field (kz much less than 1 is not satisfied). The fractional momentum spread, including second order in $\mathrm{z}$, is

$\delta_{A}\left(z_{A}\right)=r_{A} z_{A}+t_{A} z_{A}^{2}+\mathrm{O}\left(z_{A}^{3}\right)$

where $t_{A}=\frac{1}{2} \frac{d^{2} \delta_{A}}{d z^{2}}$. When the beam is compressed using magnetic compression the longitudinal coordinate becomes $\mathrm{z}_{\mathrm{a}} \rightarrow \mathrm{z}_{\mathrm{BC}}=\mathrm{z}_{\mathrm{A}}+\mathrm{R}_{56, \mathrm{BC}} \delta_{\mathrm{A}}+\mathrm{T}_{566, \mathrm{BC}} \delta^{2}{ }_{\mathrm{A}}\left(\mathrm{T}_{566, \mathrm{BC}}\right.$ is the second-order momentum compaction), and the final rms bunch length becomes

$\sigma_{z, B C}=\left[\mu^{2} \sigma_{z_{0}}^{2}+v^{2}\left\langle z_{A}^{4}\right\rangle+2 \mu v\left\langle z_{A}^{3}\right\rangle+R_{56, B C}^{2}\left\langle\delta_{A, u}^{2}\right\rangle+T_{566, B C}^{2}\left\langle\delta_{A, u}^{4}\right\rangle\right]^{1 / 2}$

where $v \mathrm{Yt}_{\mathrm{A}} \mathrm{R}_{56, \mathrm{BC}}+r_{\mathrm{A}}^{2} \mathrm{~T}_{566, \mathrm{BC}}$ and $\delta_{\mathrm{A}, \mathrm{u}}$ is the stochastic fractional momentum spread. The 
distortion imposed by the second order effects impacts the charge density profile [42] and results in a larger rms bunch length (see Fig. 33). The second order contribution on the bunch compression can be suppressed provided $\mu=0$. At medium or high energies ${ }^{4}$ two possible alternatives for canceling $\mu$ are (1) design a bunch compressor with proper $\mathrm{R}_{56, \mathrm{BC}} / \mathrm{T}_{566, \mathrm{BC}}$ ratio, or (2) locally linearize the longitudinal phase space by using a harmonic rf accelerating section operated on a decelerating phase $[44,45]$. The first solution requires a bending system that provides the same sign for $\mathrm{R}_{56, \mathrm{BC}}$ and $\mathrm{T}_{566, \mathrm{BC}}$. This requirement can be implemented e.g. with FODO-type arc that includes sextupoles, but cannot be met with a standard four-dipoles chicane. In the case of local linearization of the phase space, the harmonic rf section allows an independent control of the coefficient $r_{\mathrm{A}}$ and $\mathrm{t}_{\mathrm{A}}$ [46]. In the latter case the second order correlation coefficient $t_{A}=-e k^{2} / E_{A}\left(V_{r f} \cos \varphi+V_{r f, m} m^{2} \cos \varphi_{m}\right)$ (where $m \in N$ is the harmonic number) can be zeroed by a proper choice of the operating parameters $\mathrm{V}_{\mathrm{rf}, \mathrm{m}}$ and $\varphi_{\mathrm{m}}$ of the harmonic accelerating section. The introduction of a harmonic rf section is generally preferred since it has the advantage, compared to a taylored dispersive section, not to introduce coupling between the longitudinal and transverse phase spaces. In the case of ERLs operating at the TESLA frequency $(\mathrm{f}=1.3 \mathrm{GHz})$, a third harmonic accelerating cavity $(\mathrm{f}=3.9 \mathrm{GHz})$ has been developed at Fermilab [47] and will soon be installed in the injector [48] of the TESLA vacuum ultraviolet FEL at DESY.

If the compression is set-up to perform linearly, e.g. by using a higher order harmonic section, the final fractional momentum spread after deceleration is

\footnotetext{
${ }^{4}$ At non-relativistic energies, nonlinear compression can be used to linearize the phase space [44]
} 
$\delta_{D}=\delta_{W}\left(\frac{\varepsilon_{W}}{\varepsilon_{D}}+R_{56, R} r_{D}\right)+\delta_{W}^{2}\left(r_{D} T_{566, R}+t_{D} R_{56, R}^{2}\right) \mathrm{Y} \bar{\mu} \delta_{W}+\bar{v} \delta_{W}^{2}$

where $\mathrm{R}_{56, \mathrm{R}}$ and $\mathrm{T}_{566, \mathrm{R}}$ are the parameters associated to the recirculation transport downstream of the bunch compressor, the subscript ${ }_{\mathrm{W}}$ refers to the parameters after the wiggler, and $\mathrm{r}_{\mathrm{D}}$ and $t_{D}$ are the linear and quadratic correlations induced by the deceleration. Since the path length is properly set to have $\varphi^{\prime}=\pi+\varphi$, we have the relations $\left(\mathrm{r}_{\mathrm{D}}, \mathrm{t}_{\mathrm{D}}\right)=-\left(\mathrm{r}_{\mathrm{A}}, \mathrm{t}_{\mathrm{A}}\right) \frac{\varepsilon_{\mathrm{A}}}{\varepsilon_{D}}$. The condition $\bar{\mu}=0$ is satisfied de facto when $R_{56, B C}=-R_{56, R}$ and provided the energy loss induced by the FEL process is small compared to $\mathrm{E}_{\mathrm{A}}$. The condition $\bar{v}=0$ requires $\mathrm{T}_{566, \mathrm{R}}=-$ $t_{A} T_{566, B C}$

\subsection{Summary}

To summarize this section a presentation has been made in detail of the issues to be considered to achieve optimised longitudinal space charge profiling for the application of ERLs. This has included covering the important issue of bunch compression which for designs dependant on delivering high quality intense bunches with femto second is extremely important.

\section{SUMMARY}

The projects used to illustrate how optics issues influence design, cover a range of energies and applications at various stages and are at various stages of delivery. This has presented a wide overview of disparate issues regarding the optics design. The presentation of longitudinal dynamics issues in Section 6 illustrates how despite their differences there are a number of issues that are fundamental to ERL project design as a whole and illustrates that these can be usefully studied and understood at a more generic level. 


\section{REFERENCES}

[1] M W Poole and E A Seddon, "4GLS and the Prototype Energy Recovery Linac Project at Daresbury”, EPAC'04, Lucerne

[2] J A Clarke et al, "Prospects for a 4th Generation Light Source for the UK", PAC'01, Chicago

[3] M W Poole et al, "4GLS: an Advanced Multi-source Low Energy Photon Facility for the UK”, EPAC'02, Paris

[4] M W Poole et al, "4GLS: A New Type of 4th Generation Light Source Facility", PAC'03, Portland

[5] M. Borland, "elegant: A Flexible SDDS-Compliant Code for Accelerator Simulation ", APS LS-287, September 2000.

[6] T. Siggins et al., "Performance of a DC GaAs photocathode gun for the Jefferson lab FEL”, Nucl. Instr. and Meth. A 475 (2001) 549.

[7] A. Baeuchner et al., “The ELBE-Project at Dresden-Rossendorf”, EPAC'00, Vienna, June 2000.

[8] C. Gerth, F.E. Hannon, "Injector Design for the 4GLS Energy Recovery Linac Prototype", EPAC'04, Lucerne, July 2004.

[9] S.B. van der Geer, O.J. Luiten, M.J. de Loos, G. Poeplau, U. van Rienen “3D Spacecharge model for GPT simulations of high-brightness electron bunches'", in “Computational Accelerator Physics 2002"', edited by Berz M. and Makino K., Inst. of Physics Conf. Series No. 175, p. 101, (2005). and www.pulsar.nl

[10] K. Floettmann, “ASTRA User Manual”, September 18, 2000, http//www.desy.de/ mpyflo. 
[11] Vinokurov N. "Space Charge", in "Proceedings of the Joint US-CERN-JAPANRUSSIA Accelerator School”, St. Petersburg and Moscow, Russia (2000).

[12] B.D. Muratori, H.L. Owen, C.K.M. Gerth, S.B. van der Geer, M.J. de Loos, "Space Charge effects for the ERL Prototype Injector Line as Daresbury Laboratory”, PAC'05, Knoxville, May 2005.

[13] P. Piot, D.R. Douglas and G.A. Krafft, Phys. Rev. S.T. Accel. Beams 6, 030702 (2003)

[14] I.V. Bazarov and C.K. Sinclair, "Multivariate Optimization of High Brightness Photoinjectors", in preparation (2004)

[15] H.Padamsee et al., "An Overview of the Cryomodule for the Cornell ERL Injector", proc. of EPAC04 (2004)

[16] I.V. Bazarov, “Overview of Energy Recovery Lincas, Proceedings of PAC05 (2005)”

[17] G.H. Hoffstaetter et al., "The Cornell ERL Prototype Project”, proc. of PAC03, Portland/OR (2003)

[18] S.M. Gruner, M. Tigner (eds.), "Phase I ERL Synchrotron Light Source at Cornell University”, Report CHESS-01-003

[19] I.V. Bazarov, G.H.Hoffstaetter, "Lattice options for a 5Gev light source at Cornell”, proc. of PAC03, Portland/OR (2003)

[20] I.V. Bazarov, G.H. Hoffstaetter, “Multi-pass Beam-breakup: Theory and Calculation”, proc. of EPAC04 (2004)

[21] C. Tennant et al., "Experimental investigation of beam breakup in the Jefferson Laboratory 10kW REL” upgrade, Proceedings of PAC05 (2005)

[22] G.H. Hoffstaetter et al., "A Lattice for a 5GeV ERL in the CESR Tunnel”, proc. of PAC03, Portland/OR (2003) 
[23] I. Ben-Zvi, Ya. Derbenev, V.N. Litvinenko, L. Merminga, "Energy Recovery Linacs in High-Energy and Nuclear Physics", these proceedings

[24] http://www.physicstoday.org/vol-56/iss-10/p48.html M. Harrison et al., Special Issue of Nucl. Instrum. Methods Phys. Res. A 499 (2-3), (2003)

[25] W. MacKay, I. Ben-Zvi, J.M. Brennan, M. Harrison, J. Kewisch, S. Peggs, T. Roser, D. Trbojevic, V. Parkhomchuk "Upgrading RHIC for Higher Luminosity”, , Proc. Of PAC'2001, Chicago, Illinois U.S.A. June 18-22, 2001, http://www.agsrhichome.bnl.gov/eRHIC/

[26] D. Anderson, I. Ben-Zvi, R. Calaga, X. Chang, M. Farkhondeh, A. Fedotov, J. Kewisch, V. Litvinenko, W. Mackay, C. Montag, T. Roser, V. Yakimenko, http://www.agsrhichome.bnl.gov/eRHIC/eRHIC ZDR/AppendixA.pdf

[27] V. N. Litvinenko et al., Proceedings of the 2004 FEL Conference, p. 570, http://accelconf.web.cern.ch/AccelConf/f04

[28] V.N. Litvinenko, D. Kayran, R. Hajima, "Merger Designs for ERLs", these proceedings ERL 2005, Newport News, VA.

[29] R. Calaga et al., High Current Superconducting Cavities at RHIC, TUPKF078, Proceedings of EPAC-2004, Geneva, Switzerland, July 5-9, 2004.

[30] L. M. Young, J. H. Billen, "Parmela documentation", LA-UR-96-1835.

[31] Zero Design Report on Electron Cooling of RHIC, http://www.agsrhichome.bnl.gov/eCool/,

[32] J.Kewisch, I.Ben-Zvi, X. Chang, "Electron beam generation and transport for the RHIC electron cooler", In Proceedings of 2005 Particle Accelerator Conference, May 16-20, 2005, Knoxville. TN, USA 
[33] R. Brinkmann, Ya. Derbenev, K. Floettmann, Phys. Rev. ST Accel. Beams 4:053501, 2001

[34] J. Kewisch et al., Proceedings of the 2003 Particle Accelerator Conference, Portland, Oregon U.S.A., May 12-16, 2003, p. 2007 http:/accelconf.web.cern.ch/accelconf/p03/PAPERS/WPAE028.PDF

[35] C. Montag et al., Interaction Region Design for the Electron-Ion Collider eRHIC In Proceedings of 2005 Particle Accelerator Conference, May 16-20, 2005, Knoxville. TN, USA

[36] J. Beebe-Wang et al., Synchrotron Radiation in eRHIC Interaction Region, In Proceedings of 2005 Particle Accelerator Conference, May 16-20, 2005, Knoxville. TN, USA

[37] B. Surrow, eRHIC Detector Design Studies - Implications and Constraints on the ep(A) Interaction-Region Design In Proceedings of 2005 Particle Accelerator Conference, May 16-20, 2005, Knoxville. TN, USA

[38] C. Herandez-Garcia, et al., Proc. of FEL 2004, p. 363 (2004)

[39] H. Liu, Nucl. Instr. Meth. A 400, p. 213 (1997)

[40] A.W. Chao, Physics of collective beam instabilities in high energy accelerators, J. Wiley \& Sons Ed.

[41] D.R. Douglas et al., Proc. of PAC 2003, p. 213 (2003)

[42] R. Li, Nucl. Instr. Meth. A 475, p.498 (2001)

[43] G.A. Krafft, Proc of Micro bunches Workshop, AIP Conf. Proc. No. 367, p. 46 (1996)

[44] T. Smith, Proc of LINAC 1984, SLAC report 303 (1986)

[45] Dowell, et al., Nucl. Instr. Meth. A375, p. 108 (1996)

[46] K. Floettmann, et al., DESY report TESLA-FEL-2001-06 (2001)

[47] N. Solyak, et al., Proc. LINAC 2004, p.797 (2004) 
[48] K. Floettmann and P. Piot, Proc. EPAC 2002, p. 1798 (2002) 


\section{ACKNOWLEDGEMENTS}

The Section on the 4GLS and ERLP projects at Daresbury represent the work of very many project staff at Daresbury. We have benefited from many UK and international advisors to the project. Work is funded by CCLRC and the UK Office of Science and Technology.

The Work reported under Section 5, Optic Issues of ERL Projects at BNL was performed under the auspices of the U.S. Department of Energy and partially funded by the US Department of Defense. \#vl@bnl.gov. 


\section{FIGURE CAPTIONS}

Figure 1. Illustration of some of the ongoing ERL based projects.

Figure 2. Baseline layout scheme for 4GLS.

Figure 3. Lattice functions through a TBA cell in the 150 degree high current arc.

Figure 4. Schematic of the timing structure through the 4GLS linac.

Figure 5. Layout of the ERLP injector and evolution of the beam size, norm. emittance and bunch length (all rms).

Figure 6. Transverse beam sizes, divergences and normalized emittance for a Gaussian distribution with space charge for GPT, together with the analytic estimate, for the injector model including dipoles.

Figure 7. Present layout of the ERLP.

Figure 8. Beta functions for the ERLP.

Figure 9. Close up of beta functions through the wiggler for the ERLP.

Figure 10. Dispersion for the ERLP.

Figure 11. An ERL in an extended CESR tunnel.

Figure 12. Sketch of a cross section of a tunnel with two linacs.

Figure 13. Beta functions and dispersion in the arcs.

Figure 14. Effective emittance along the arc.

Figure 15. Optics of the return loop.

Figure 16. CSR emittance growth in the return loop. Solid: with CSR, dashed: without radiation. Units are \% of $0.1 \mathrm{~nm}$ along the linac (in $\mathrm{m}$ ).

Figure 17. Optics in the two linacs (units are $\mathrm{m}$ ).

Figure 18. An ERL in the extended CESR tunnel minimizing bend angles

Figure 19. The beamline connecting the linac and the CESR ring. Undulator beam lines are shown, but a CW FEL could also operate in this region. 
Figure 20. Beta functions and dispersion for the region between the linac and CESR (units are in $\mathrm{m})$

Figure 21: Layout of the R\&D ERL.

Figure 22: Drawing of the R\&D ERL loop.

Figure 23: Lattice $\beta$ and D functions of the R\&D ERL for the case of zero longitudinal dispersion $\mathrm{D}_{\mathrm{s}}=\mathrm{R}_{56}$.

Figure 24. Evolution of the RMS beam sizes in the R\&D ERL with charges of $1.4 \mathrm{nC}$ and 10 $\mathrm{nC}$ per bunch.

Figure 25. Layout of the RHIC cooler

Figure 26. Optics of the matching section between the cooling solenoids

Figure 27. A stand alone ERL for eRHIC.

Figure 28. Lattice of achromatic arc for a stand-alone ERL.

Figure 29. Schematic of the arcs (rectangles) for the linac-ring eRHIC located in the RHIC tunnel on the top of each other. The nominal number of arcs is two for $20 \mathrm{GeV}$ case.

Figure 30. Round electron beam from ERL with an initial transverse RMS emittance of 3 $\mathrm{nm} * \mathrm{rad}$ passes through the IP with the disruption parameter 3.61 [3] (tune shift of 0.6). The interaction with the hadron beam modifies the optic functions of electron beam significantly. The dashed line is effective density of the hadron bunch and the solid line in the size of the electron beam envelope as a function of coordinate $\mathrm{z}$ around the IP.

Figure 31. Illustration of the possible phase choices for deceleration. The beam is accelerated off-crest (a), and presented at the entrance of the linac after an isochronous recirculation transport (b) and a reflecting transport (c). The red line represent the bunch w.r.t. the rf-wave (tail is on the right in our sign convention).

Figure 32. Fractional momentum spread of a $60 \mathrm{pC}$ bunch measured in the middle of the two $180^{\circ}$ arcs of the IR-Demo [measurement by P. Piot (July 1999)]. The origin of the horizontal axis is arbitrary.

Figure 33. Longitudinal phase space after compression without (a) and with (b) linearization of the longitudinal phase space prior to the bunch compressor. The profile (c) and (e) respectively corresponds to the charge density associated to (a) and (b) 
- JLAB-FEL

- JAERI-FEL

- RECUPERATOR -FEL

- ERLplus

- Cornell X-Ray ERL

- 4GLS

- MARS

- APS-ERL

- Arc en Ciel

- eCool-RHIC

- eRHIC-ERL

- ELIC

- ...

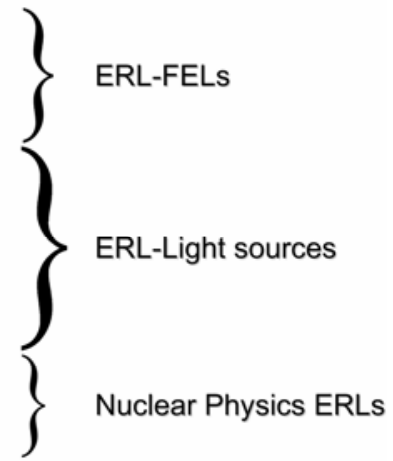

Figure 1 


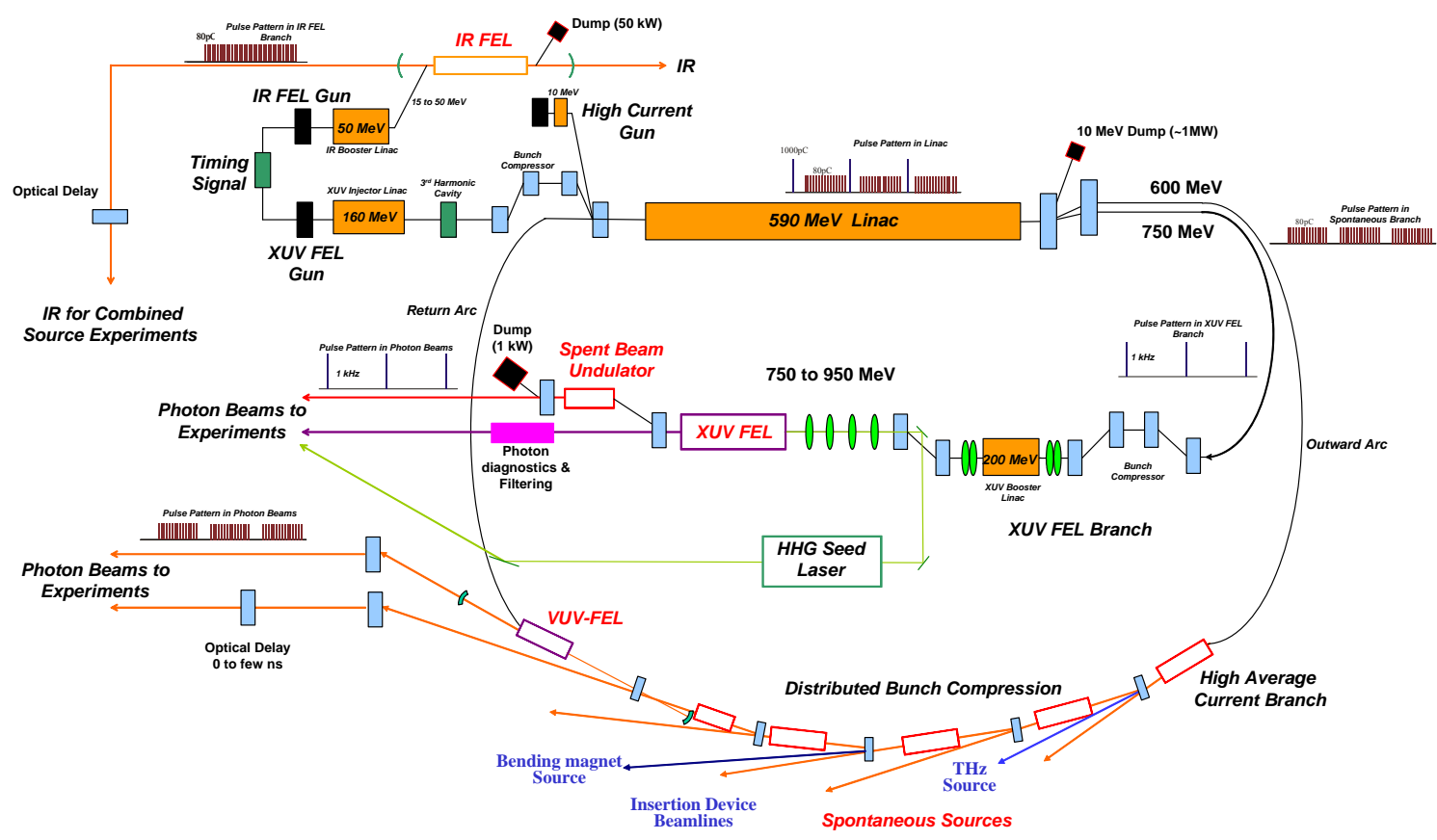

Figure 2 


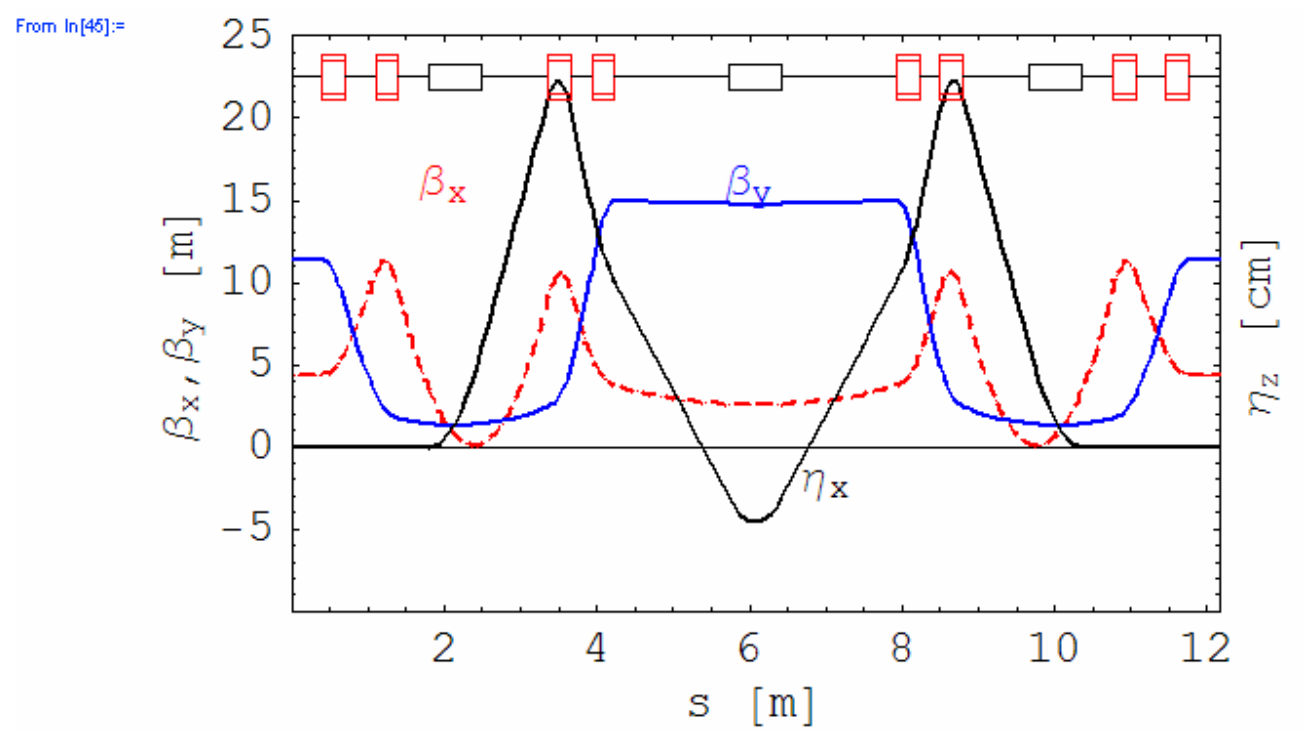

Figure 3 


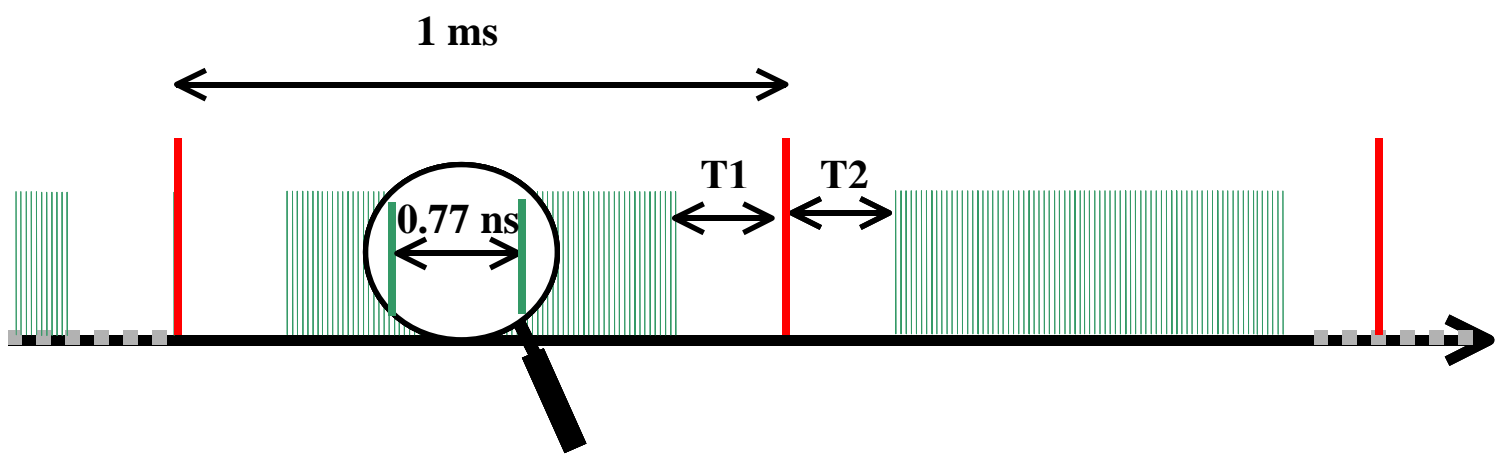

Figure 4 


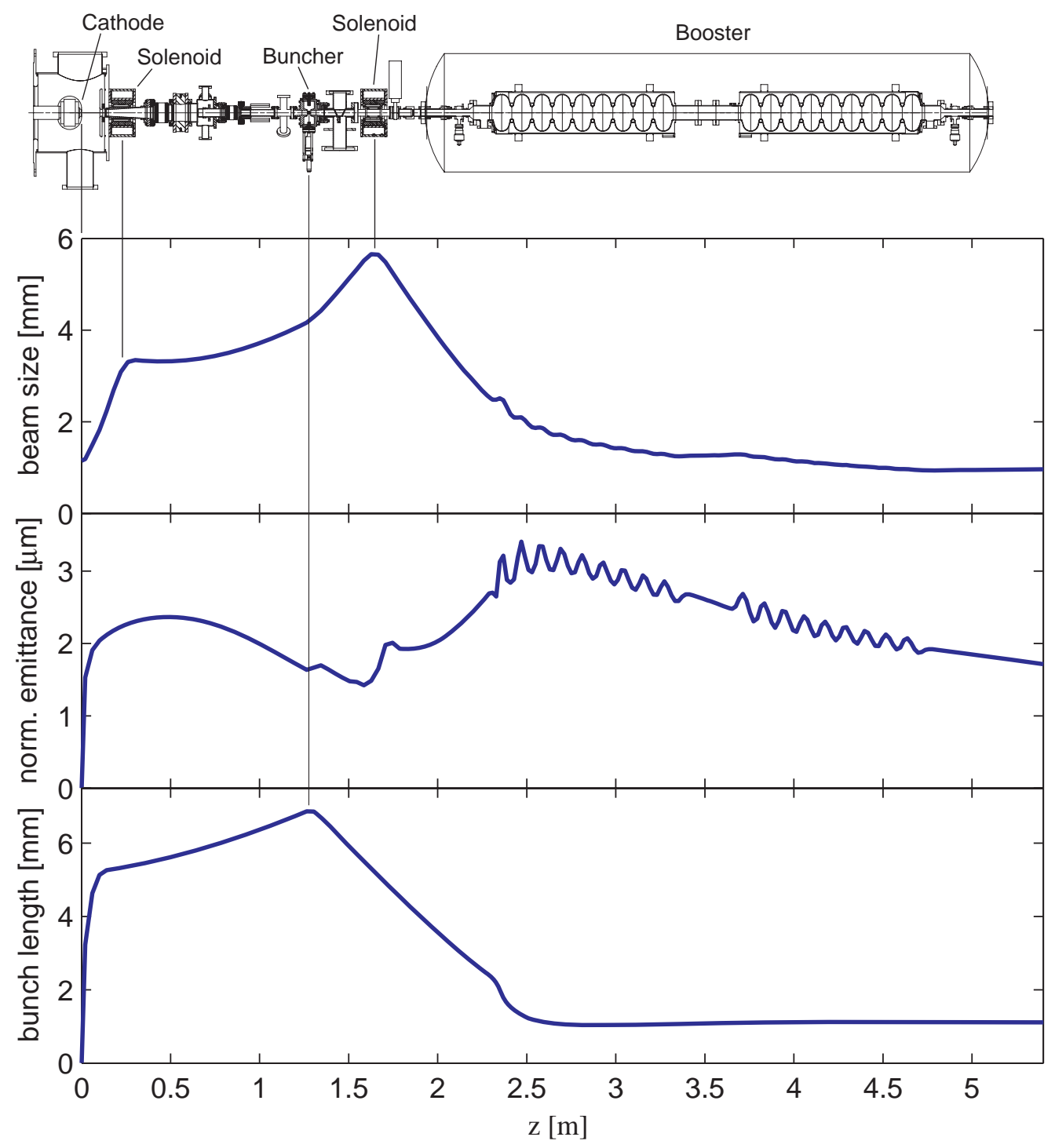

Figure 5 

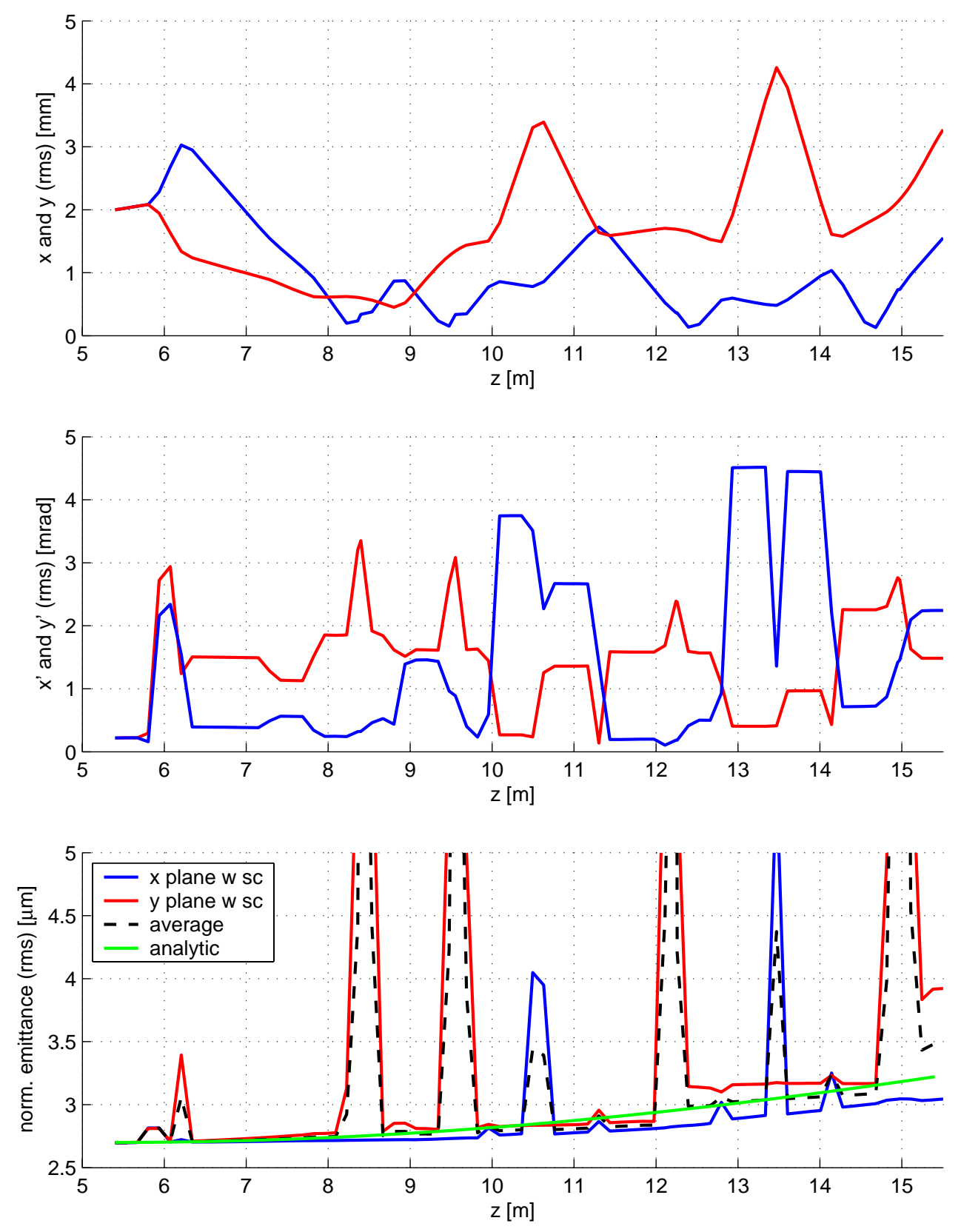

Figure 6 


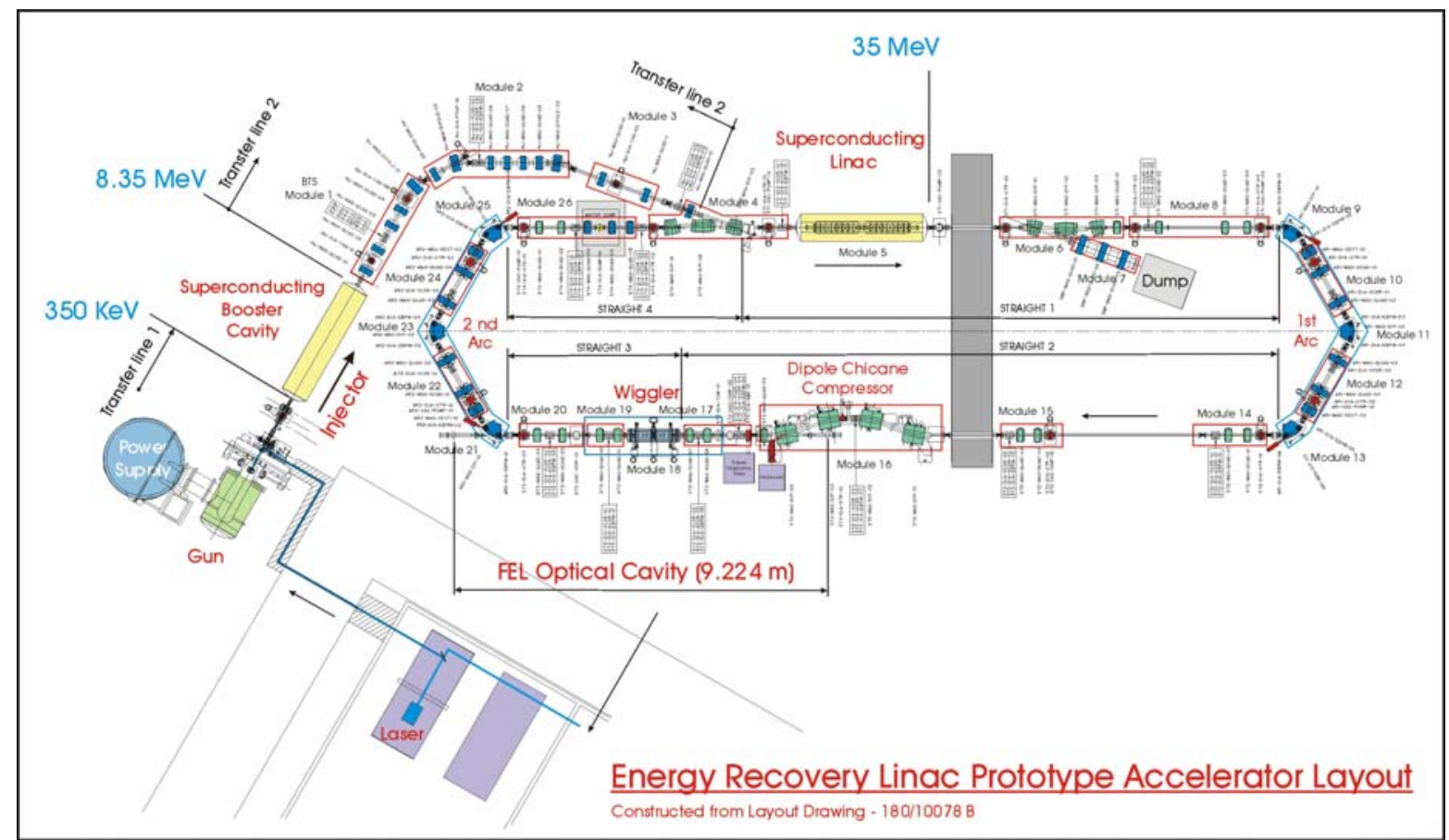

Figure 7 


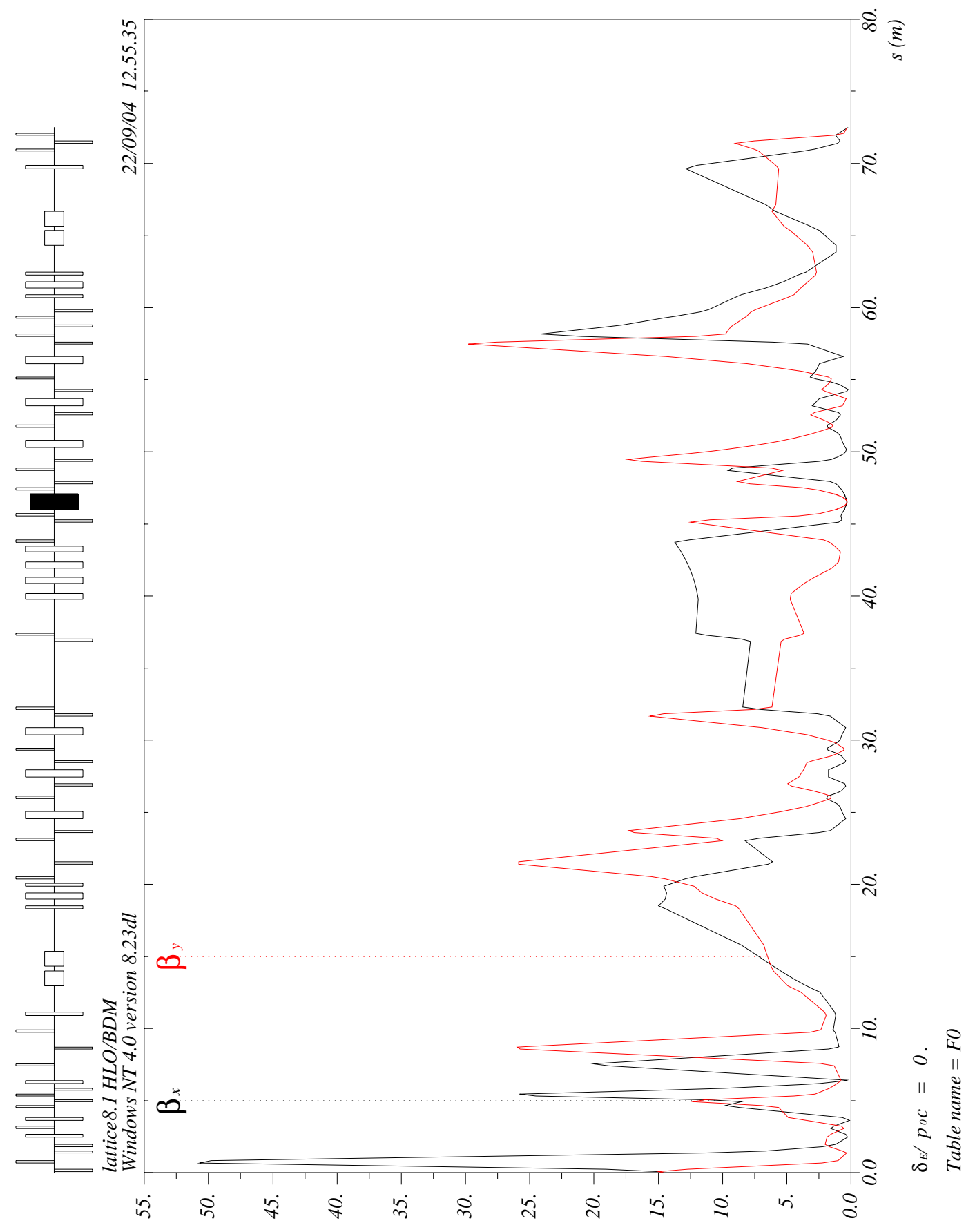

(ui) $\mathrm{g}$

Figure 8 


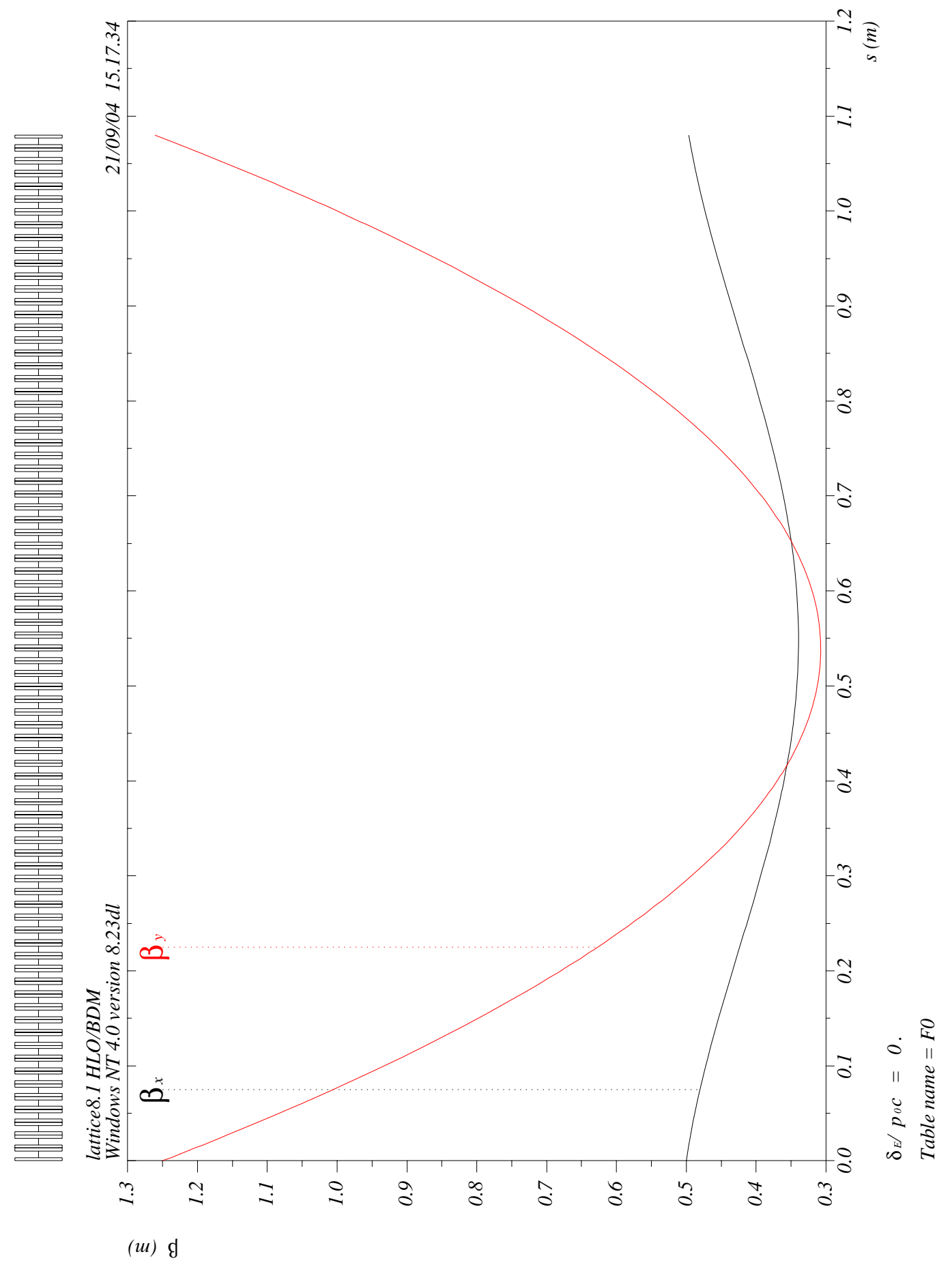

Figure 9 


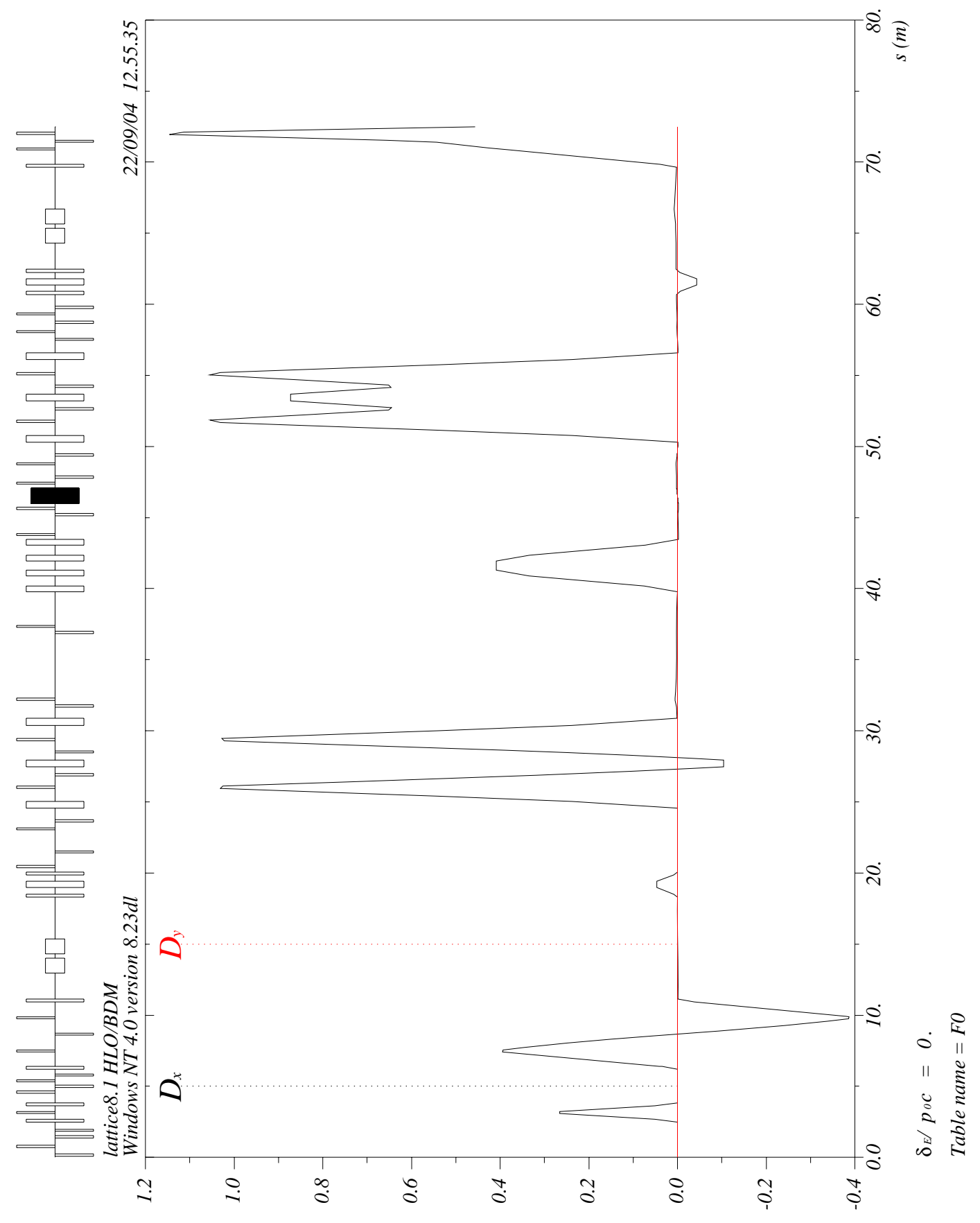

(ui) $a$

Figure 10 


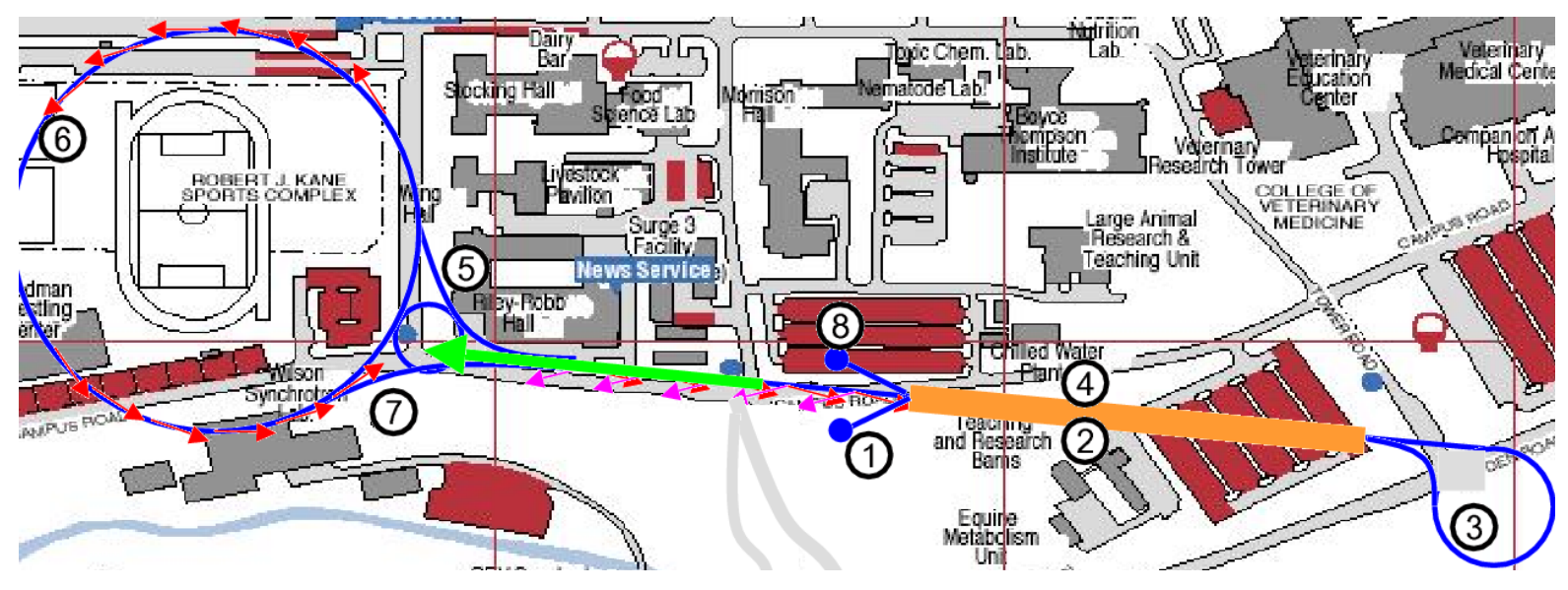

Figure 11 


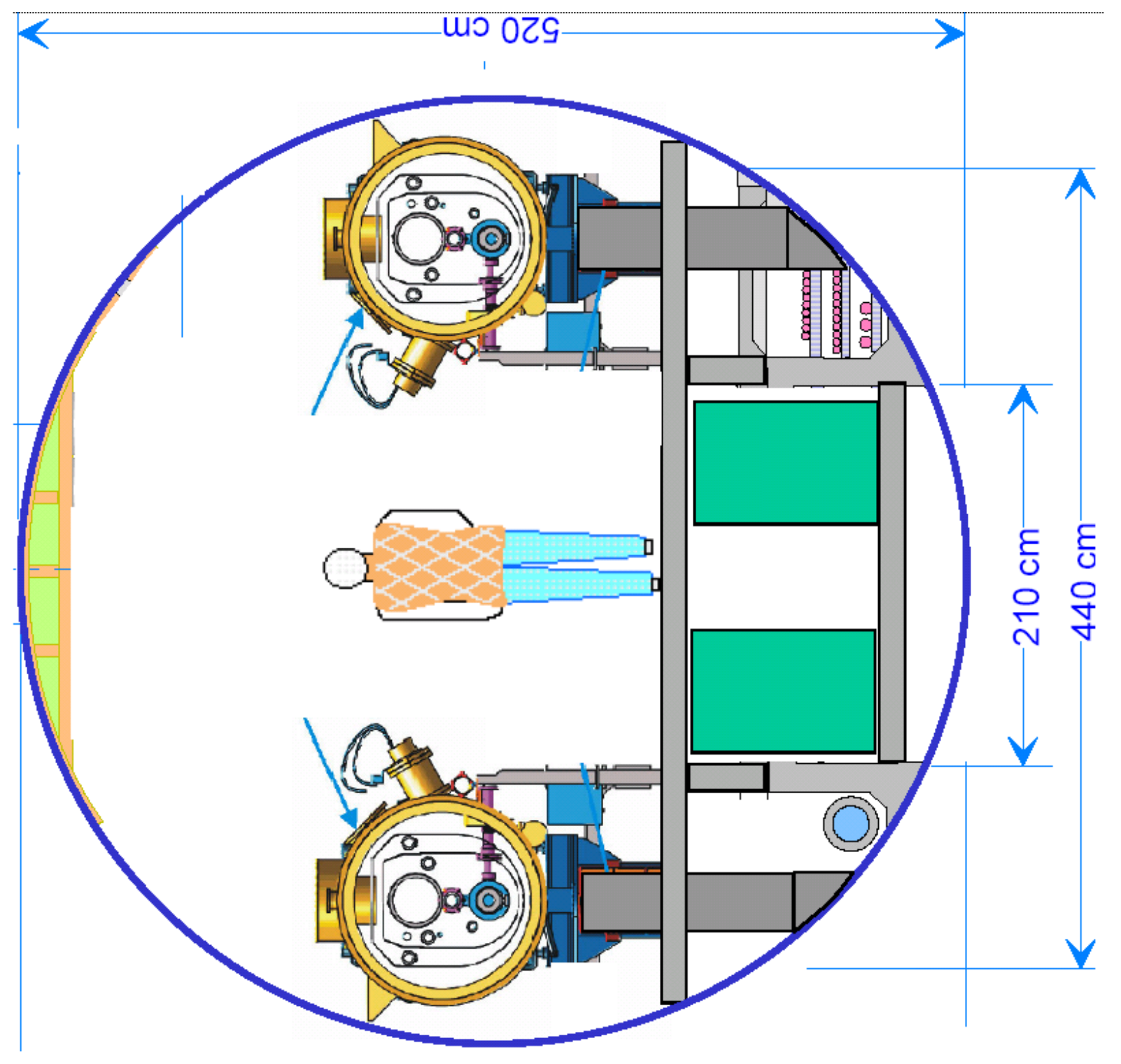

Figure 12 


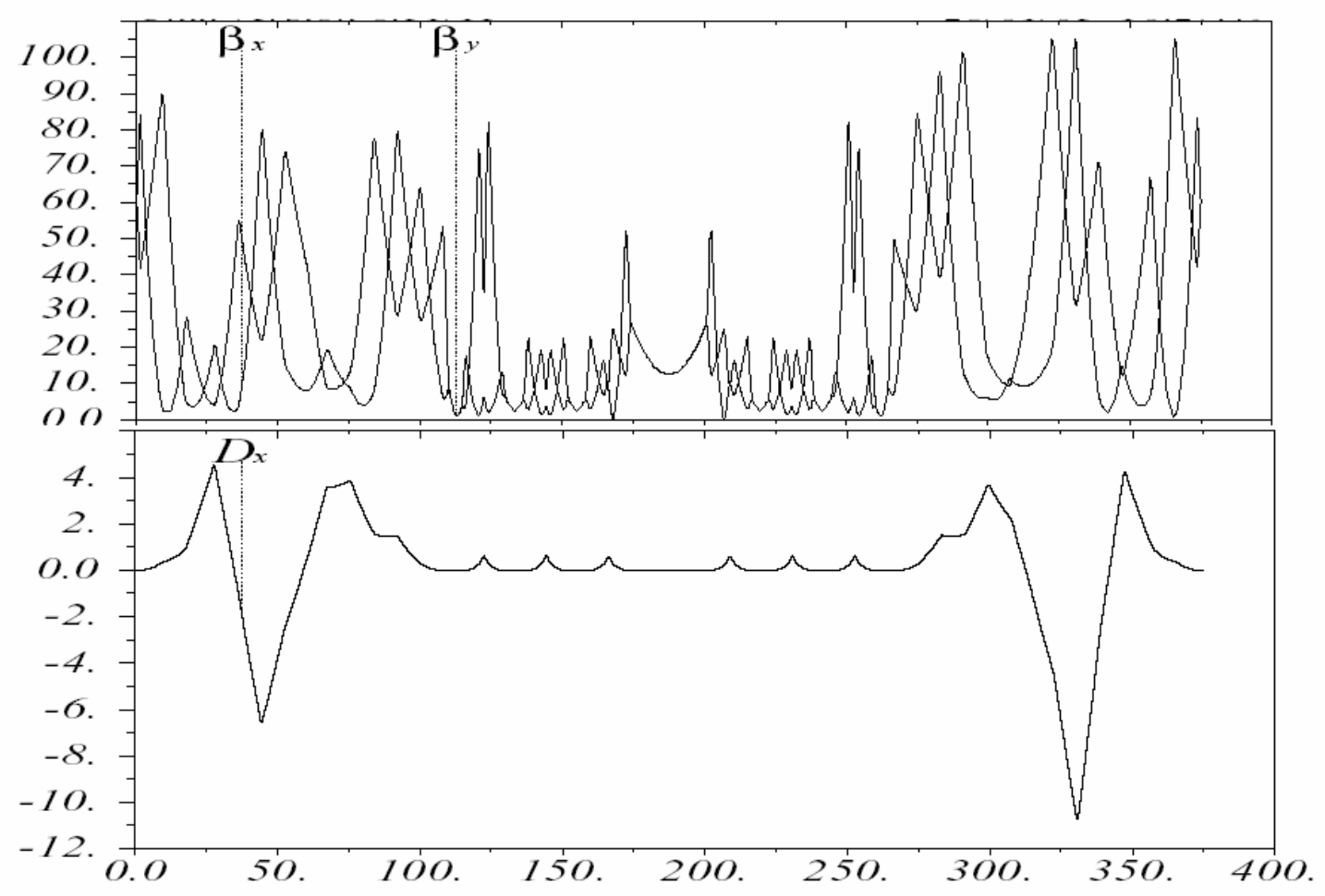

Figure 13 


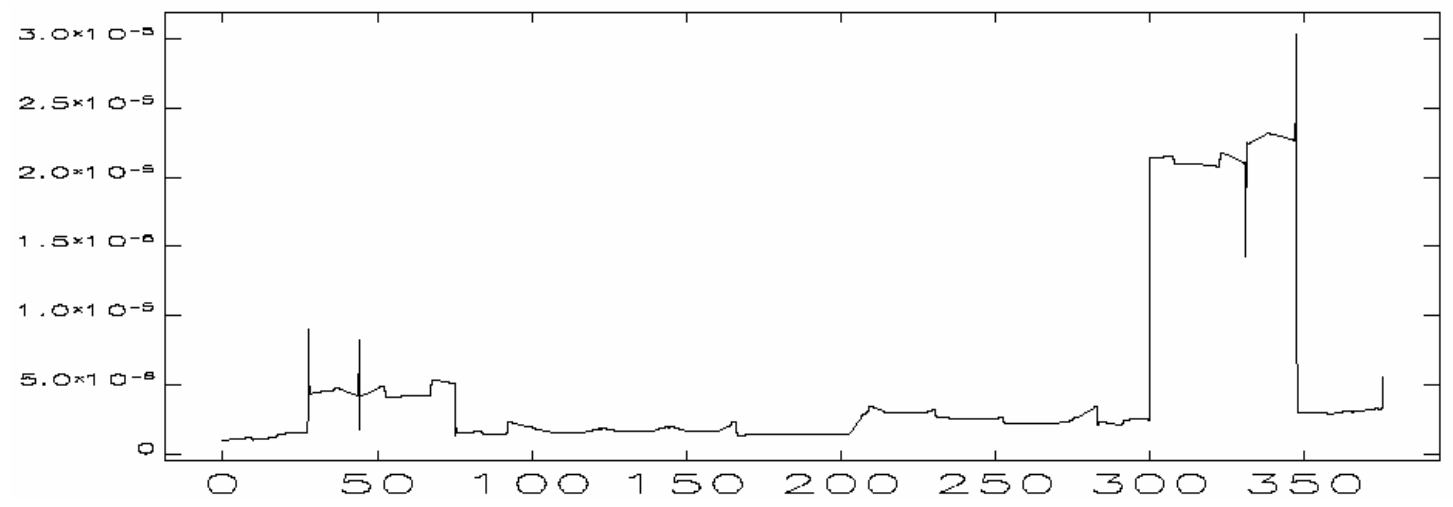

Figure 14 


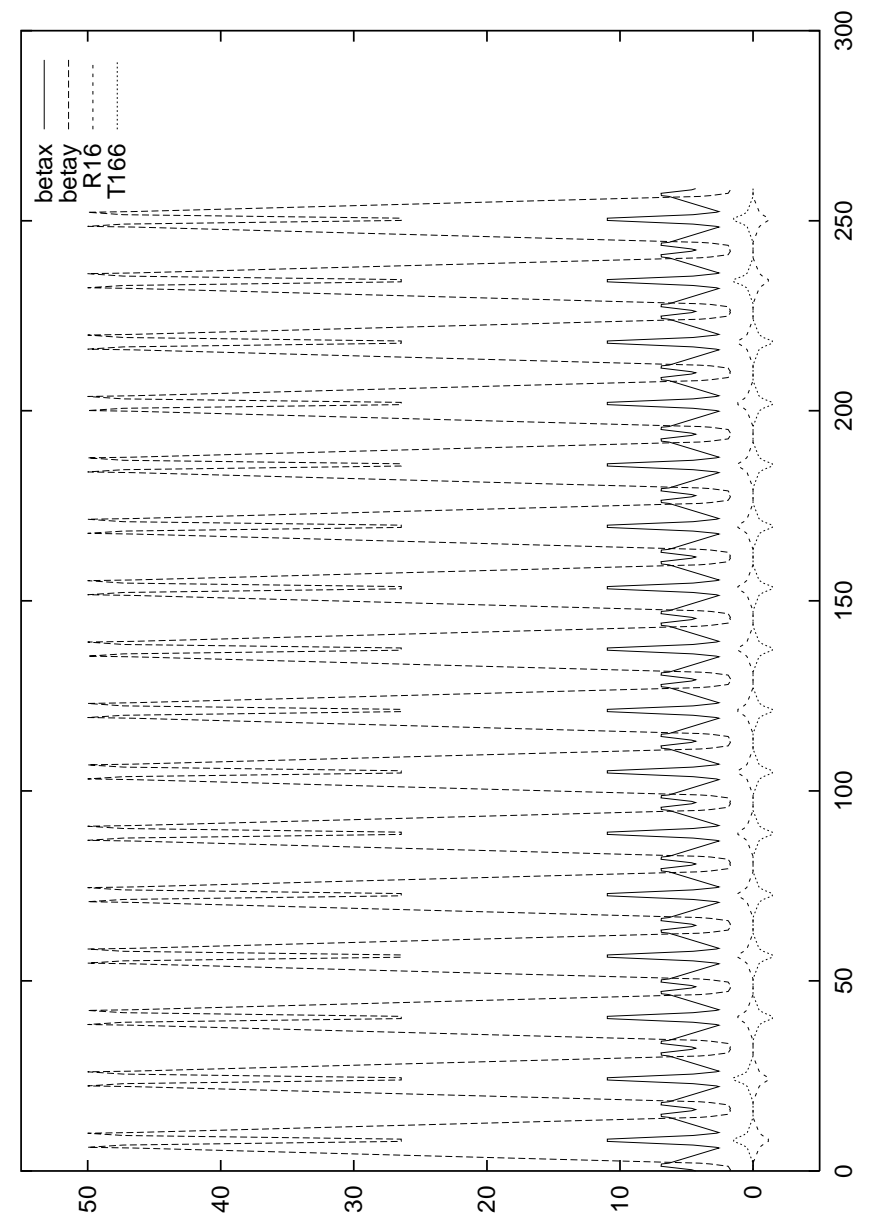

Figure 15 


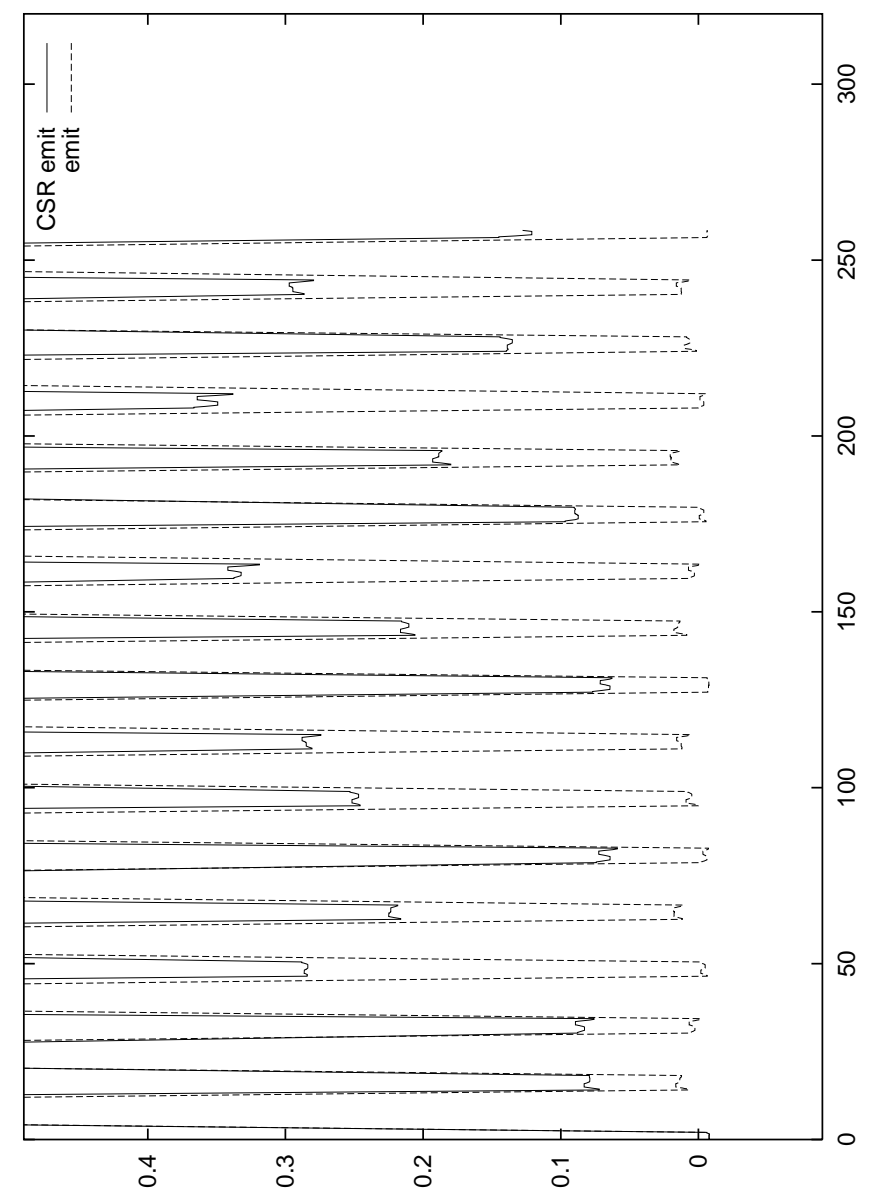

Figure 16 


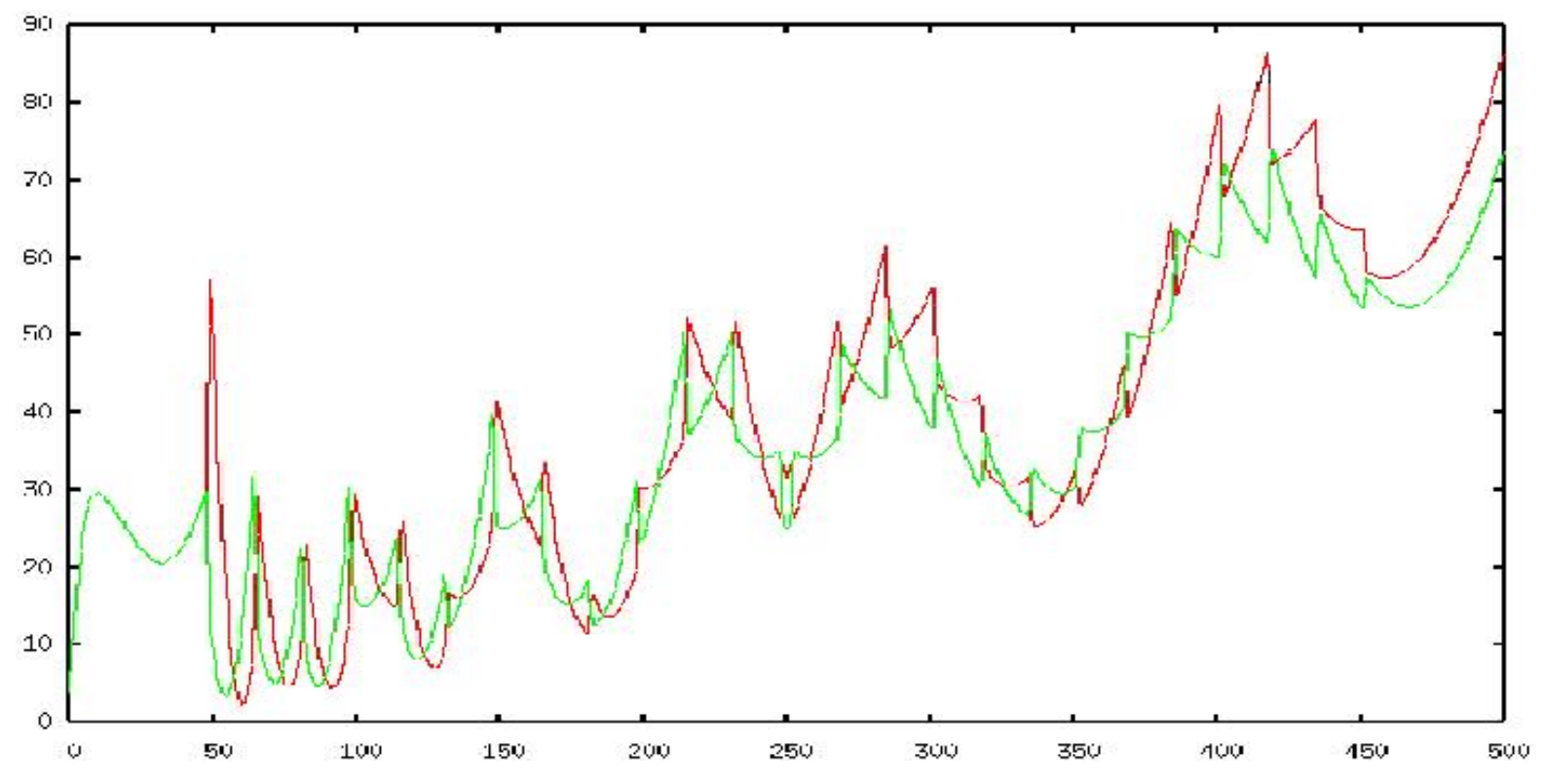

Figure 17 


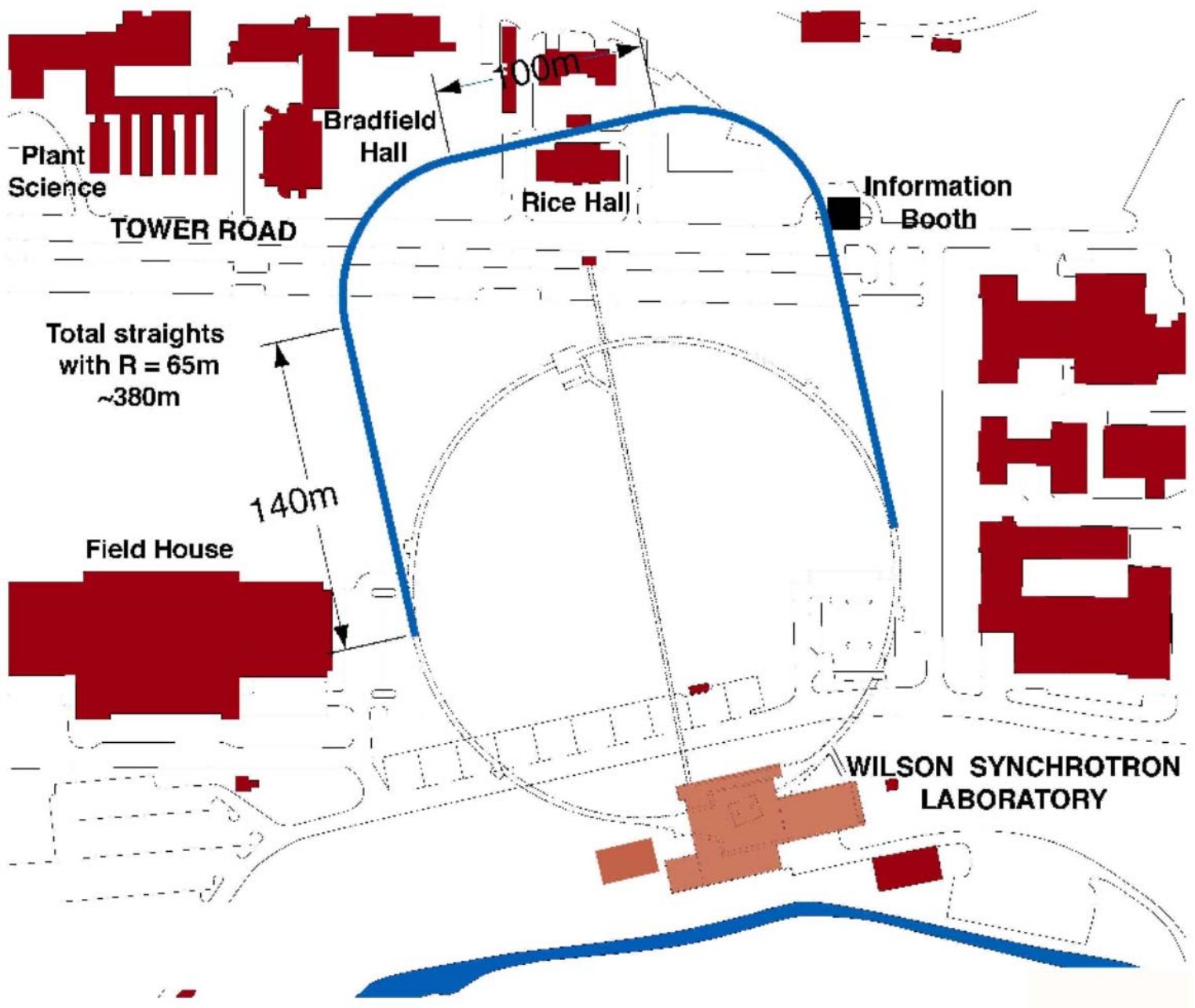

Figure 18 


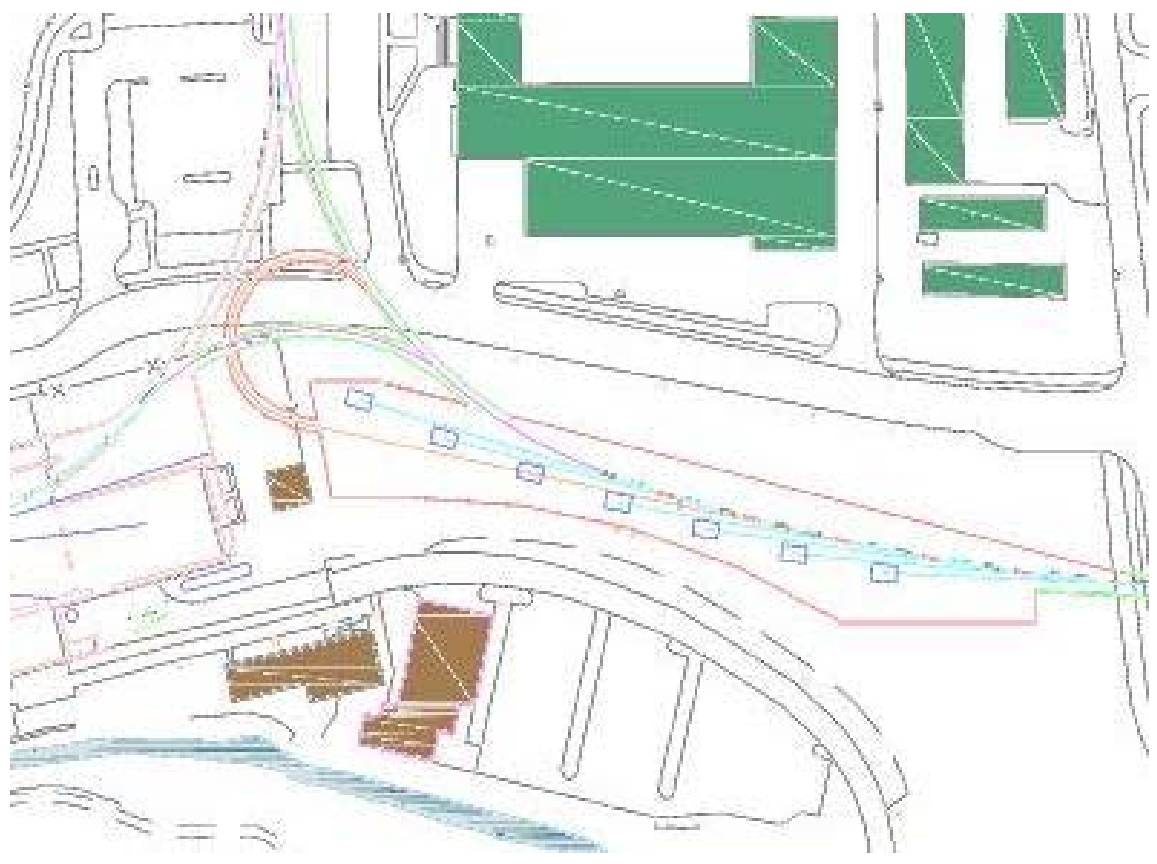

Figure 19 


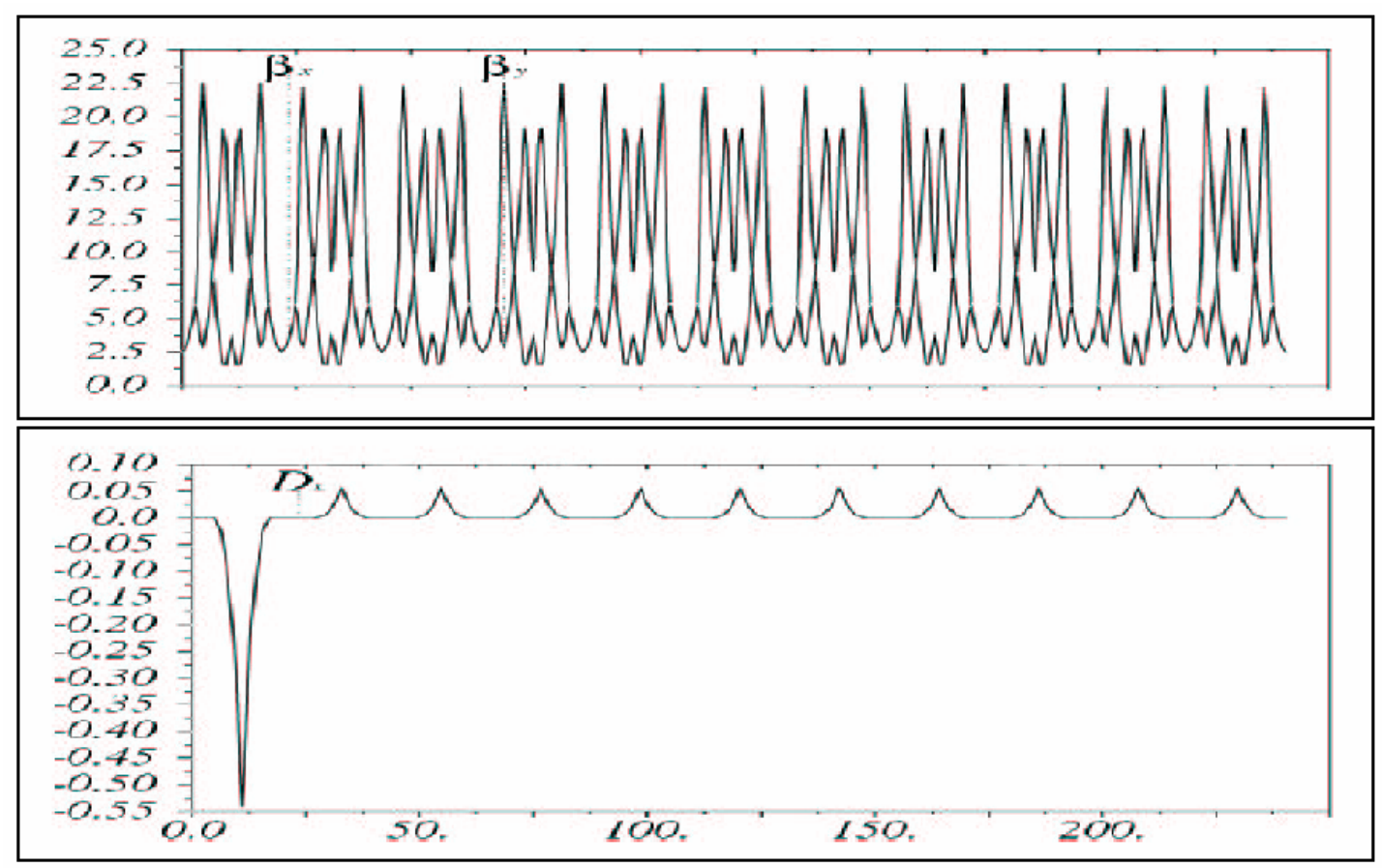

Figure 20 


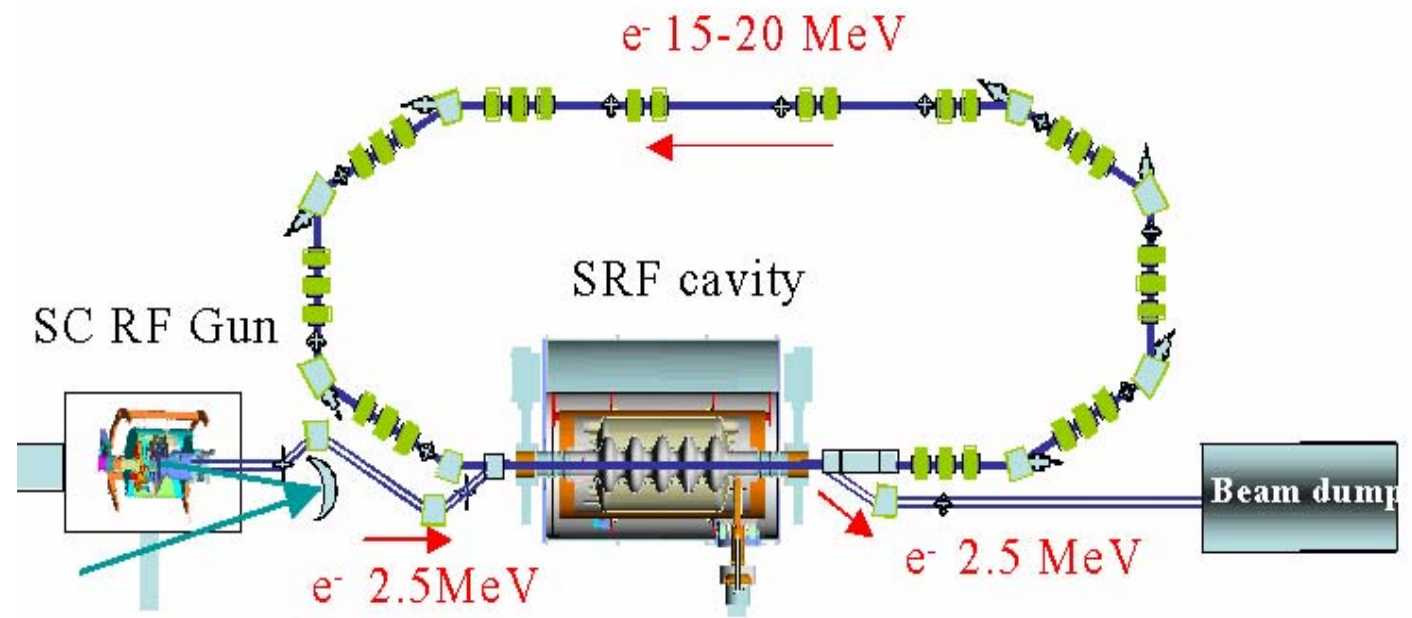

Figure 21 


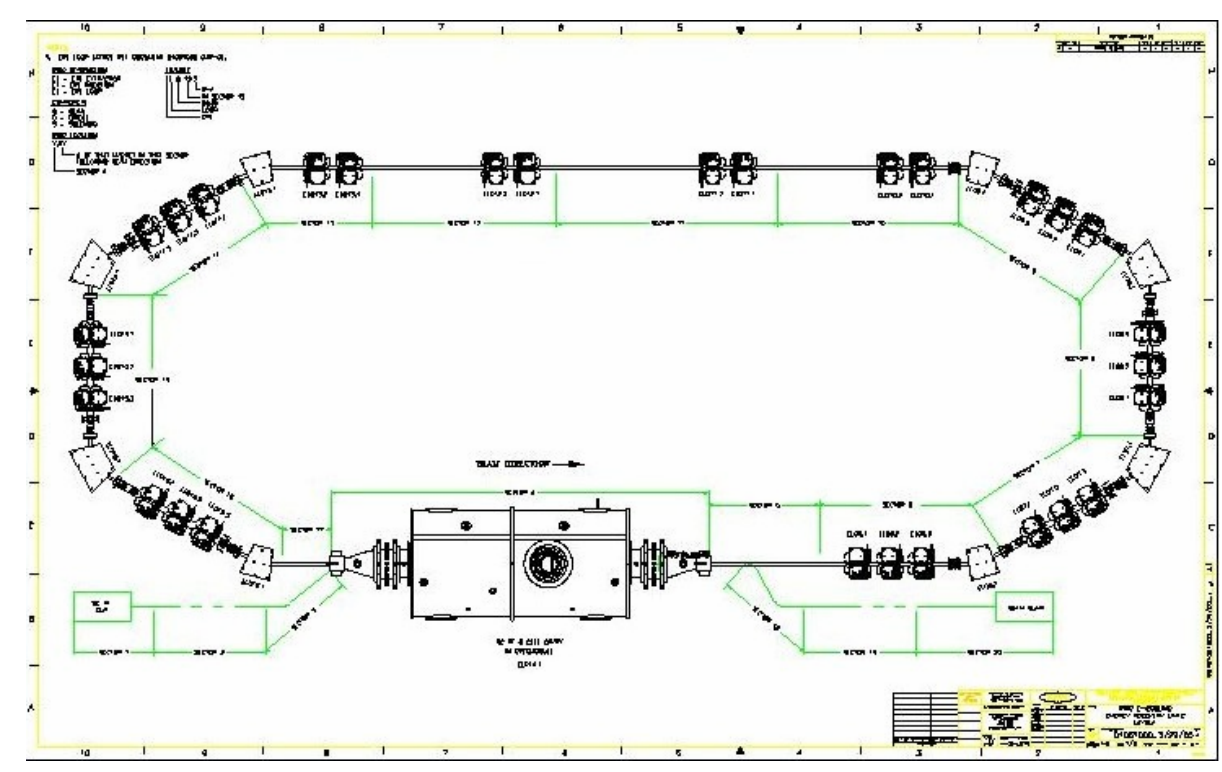

Figure 22 


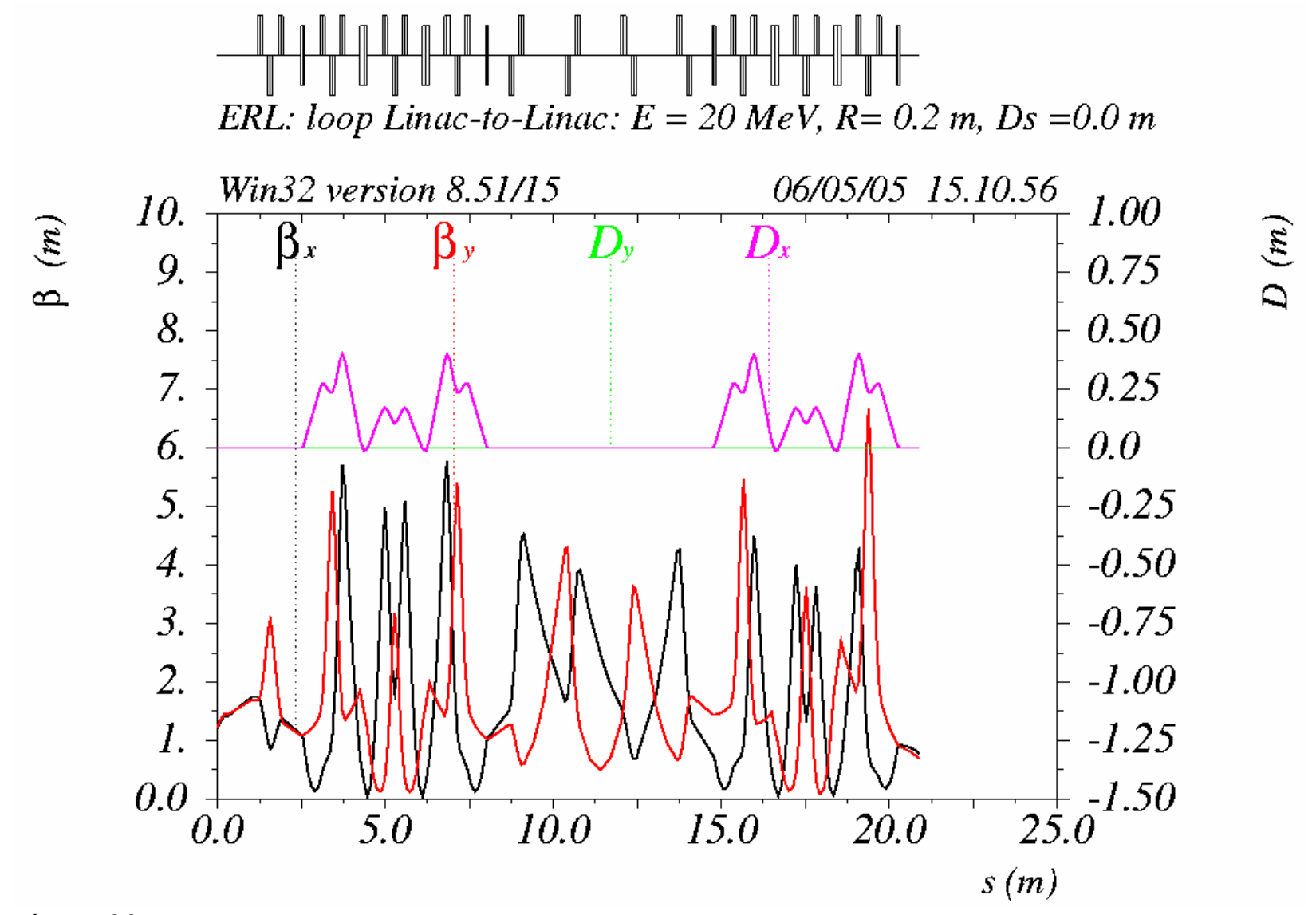

Figure 23 


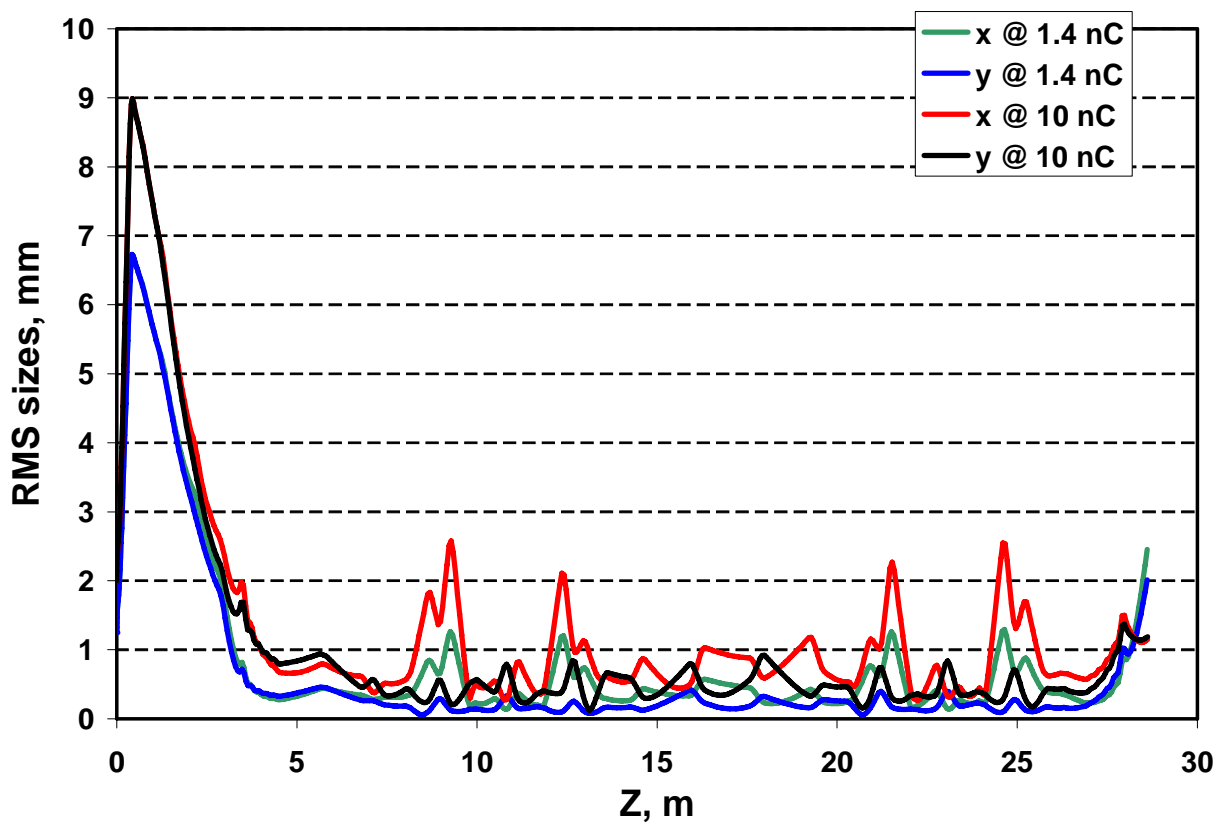

Figure 24 


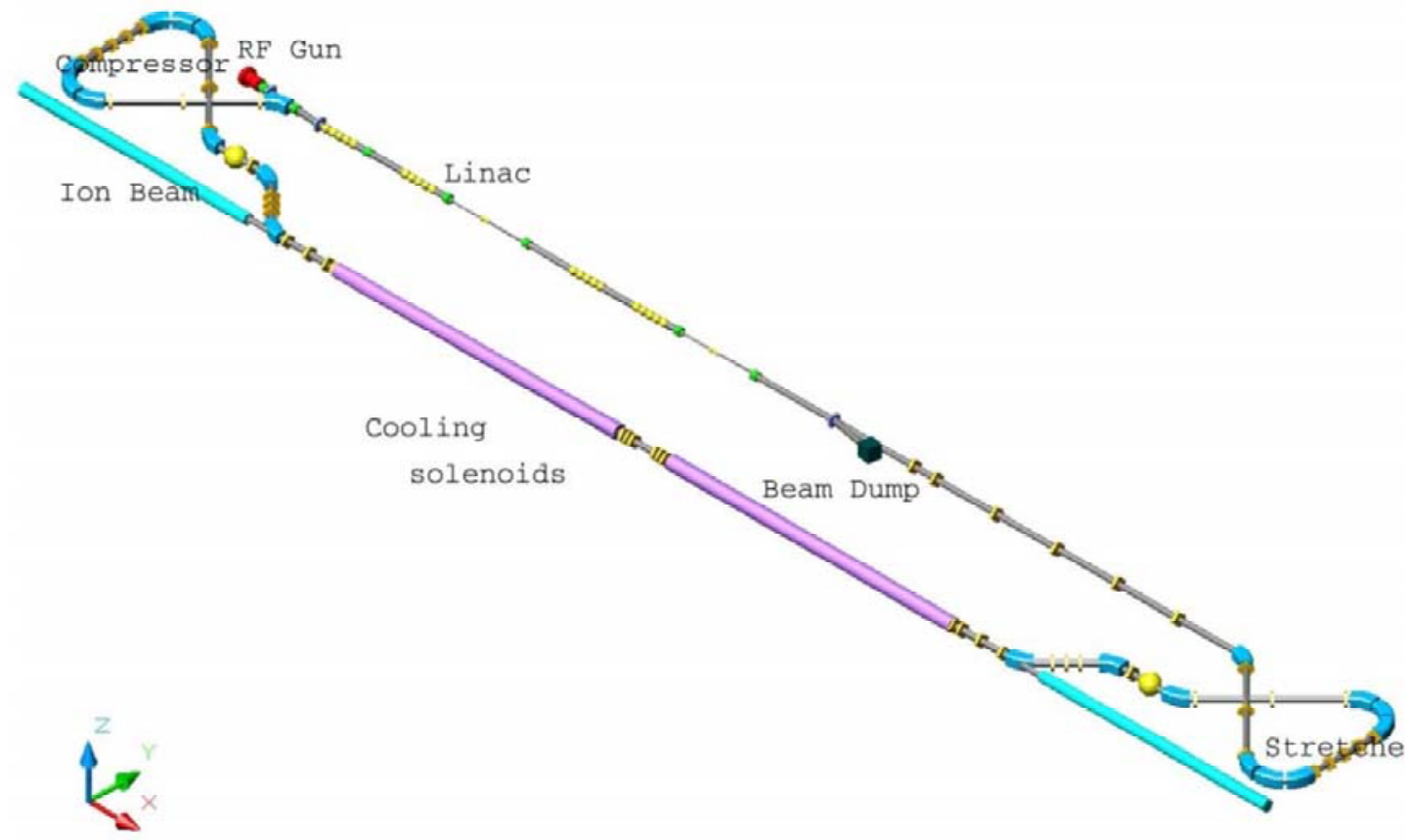

Figure 25 


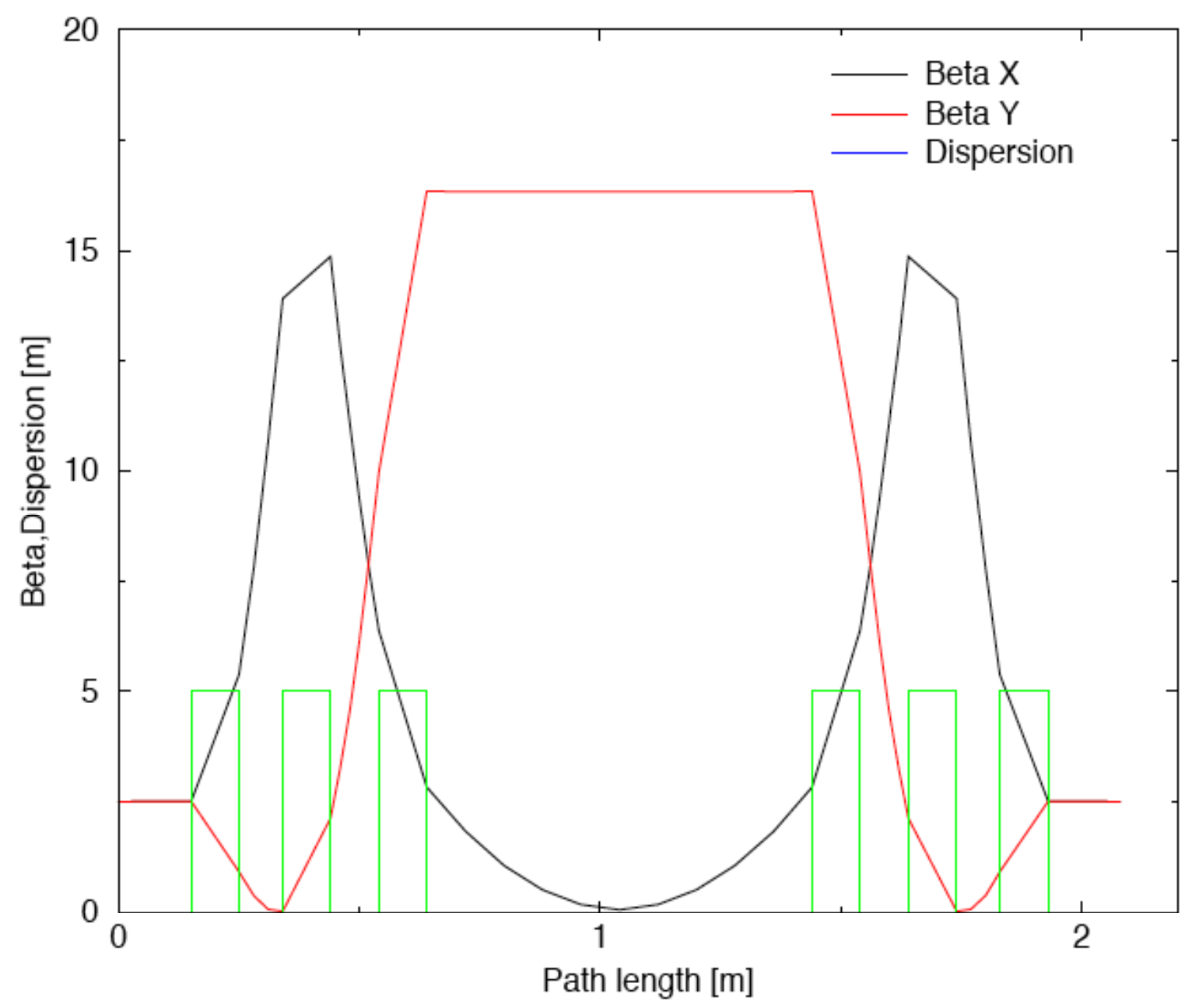

Figure 26 


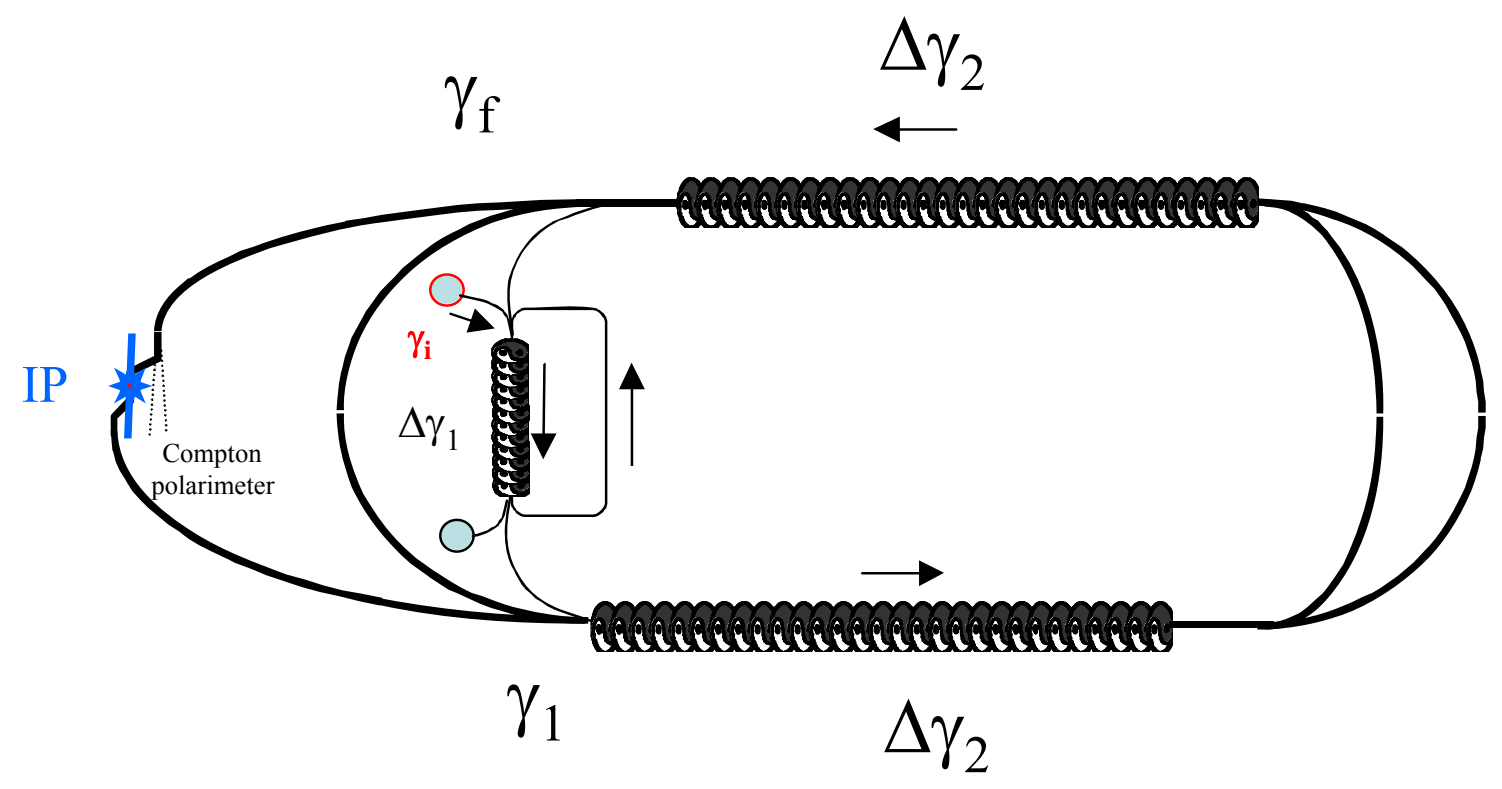

Figure 27 


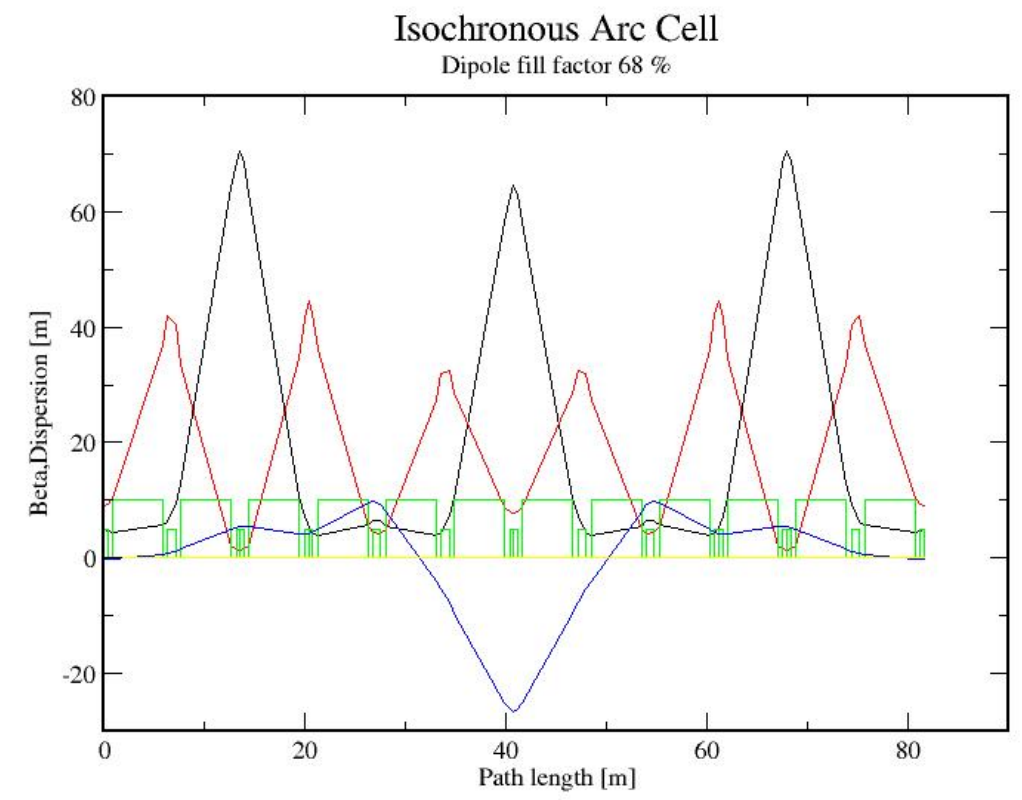

Figure 28 

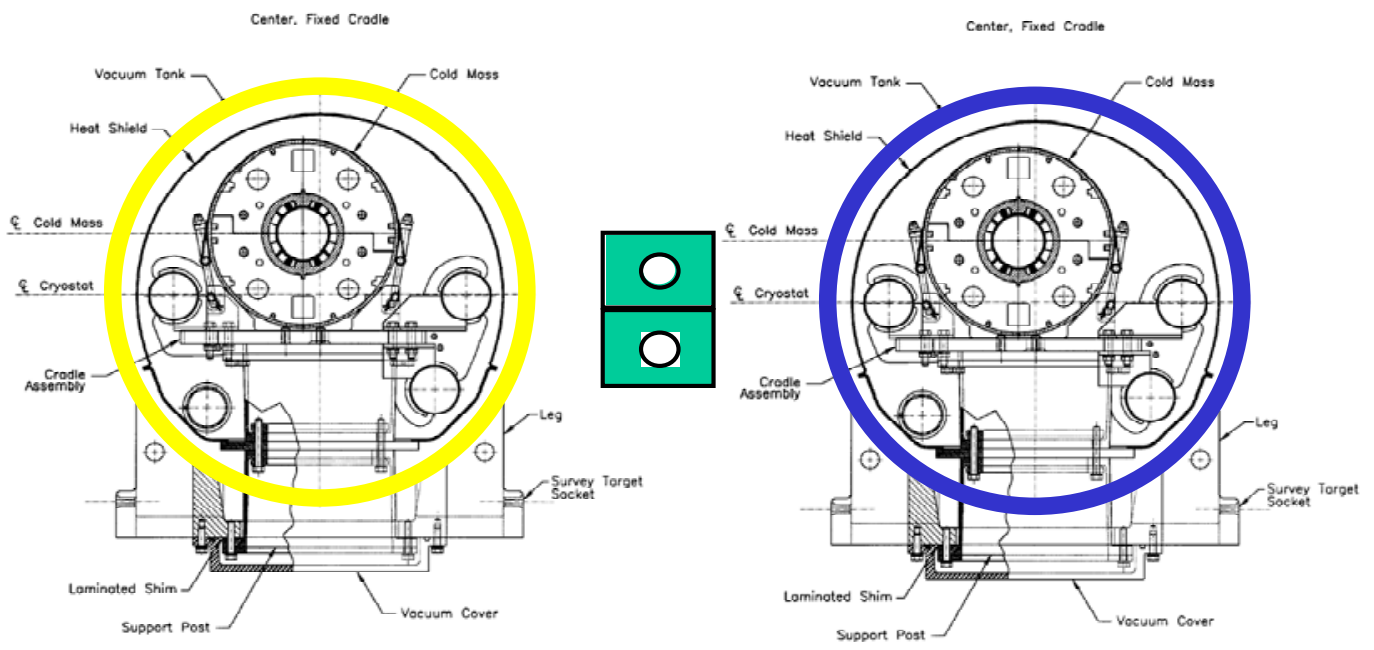

Figure 29 


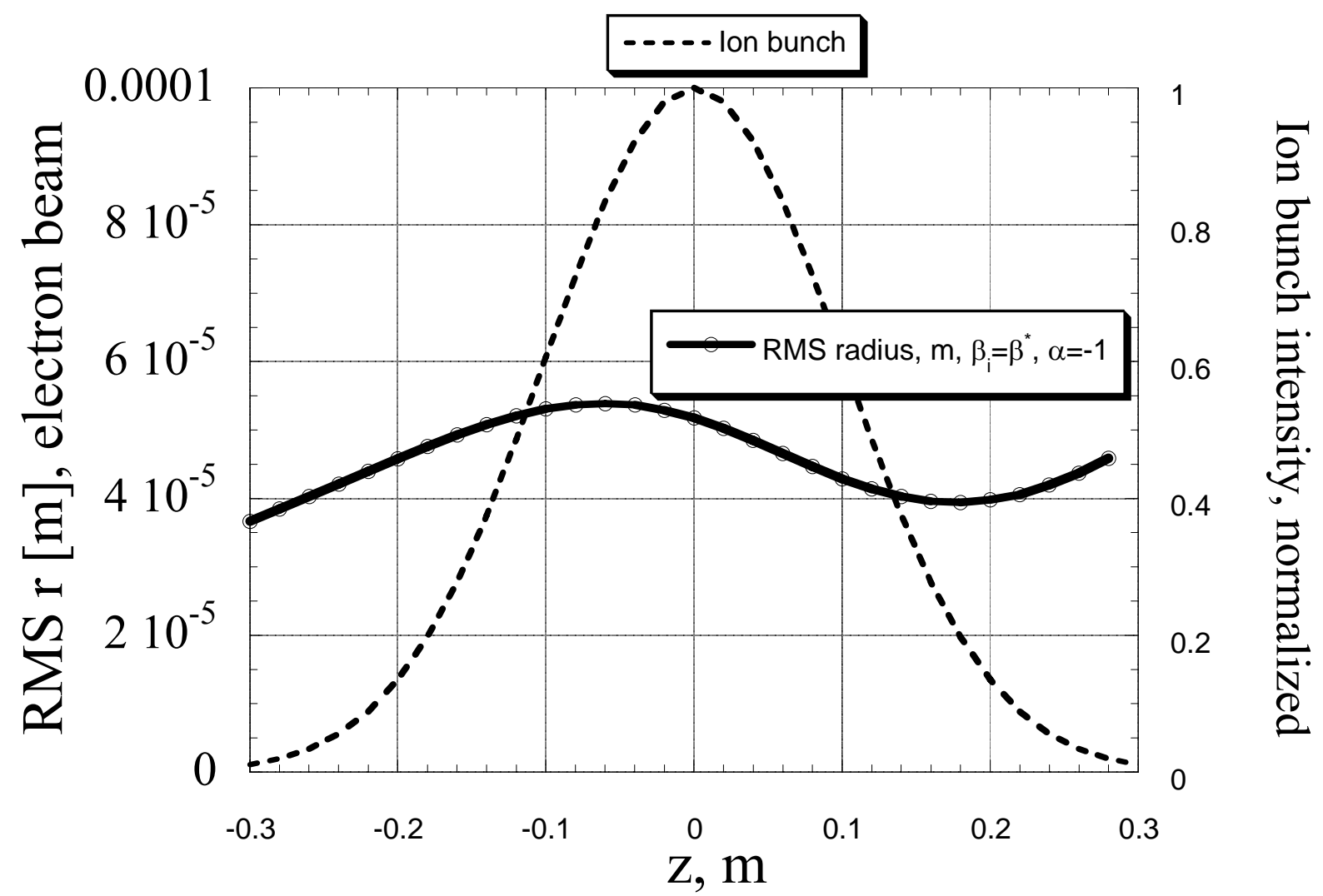

Figure 30 


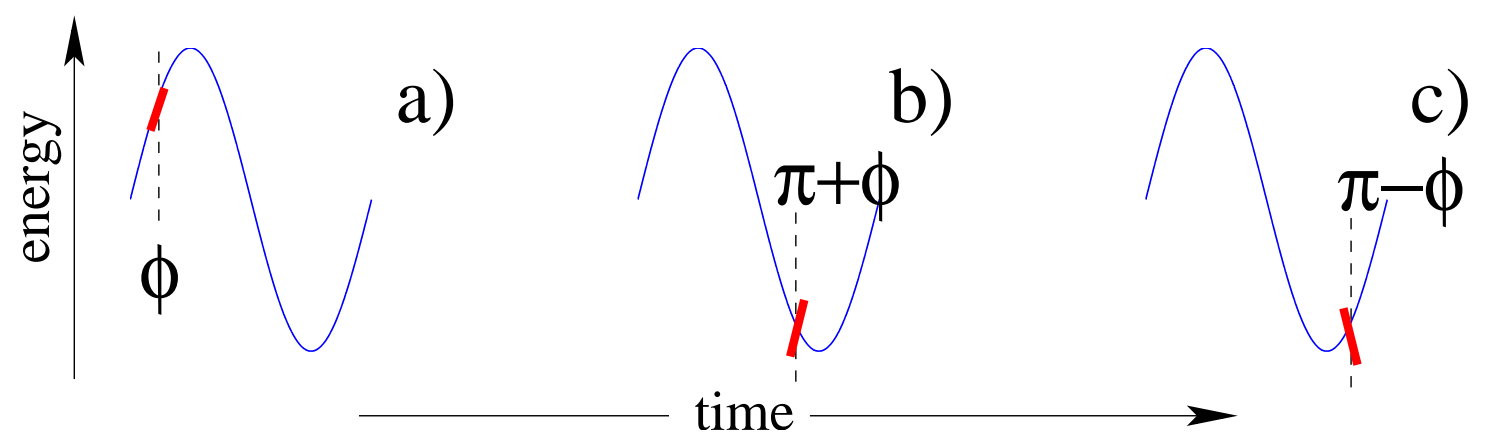

Figure 31 


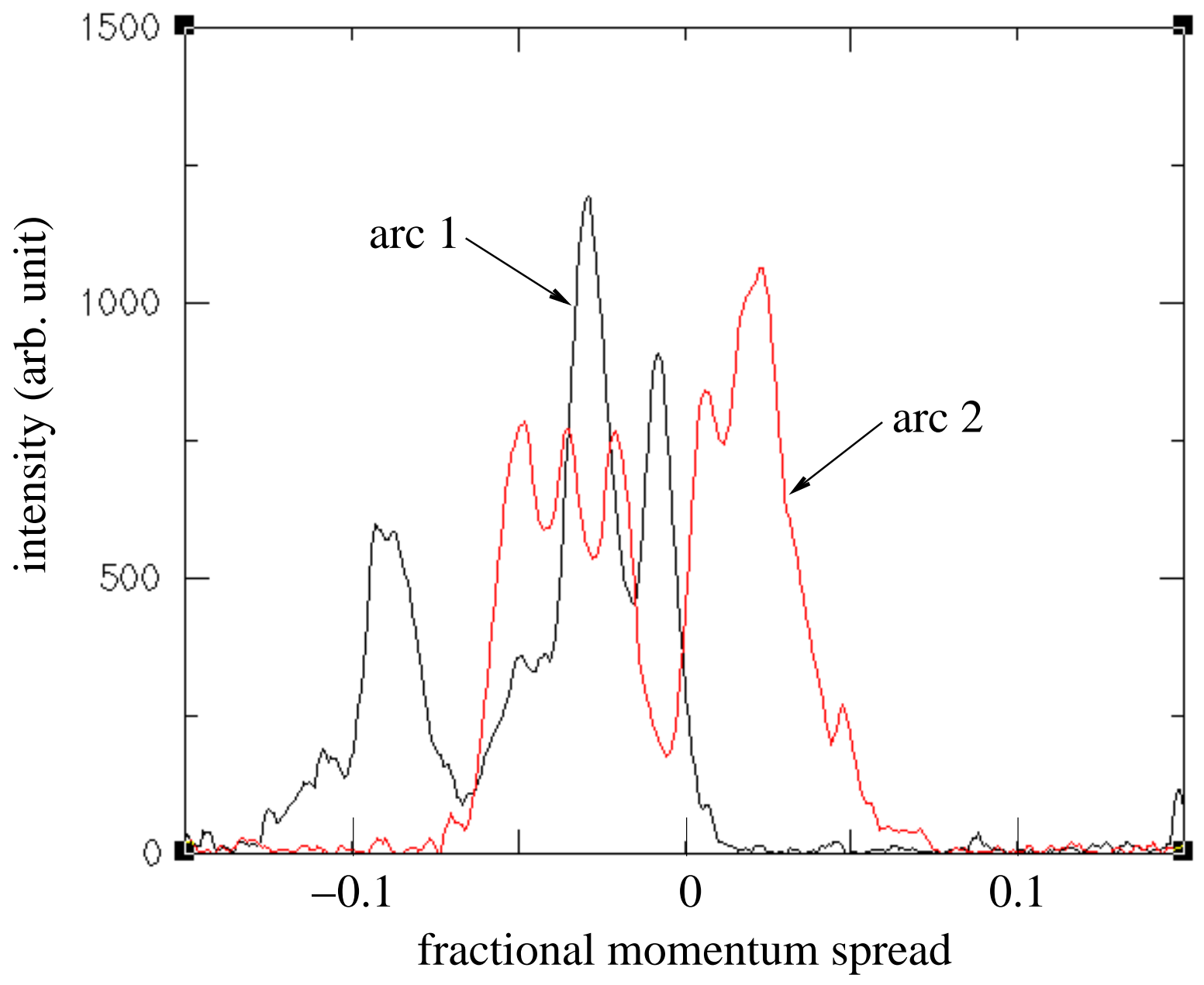

Figure 32 

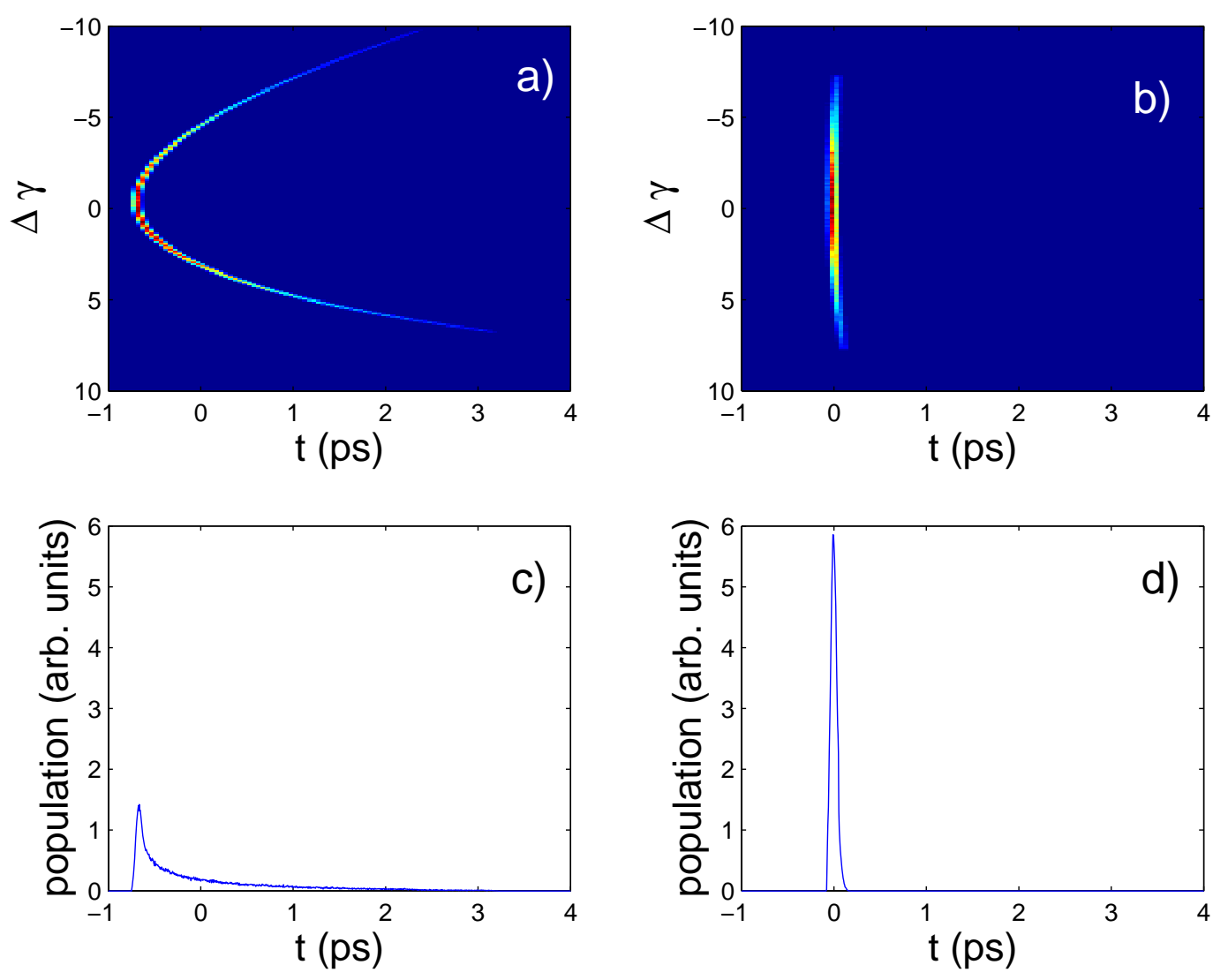

Figure 33 


\section{Tables:}

Table 1. Parameters for an ERL at Cornell University for three different running modes: for high flux, for high coherence and for short pulses. We show initial target emittance figures, simulations suggest that lower values may be possible.

\begin{tabular}{|c|c|c|c|}
\hline Current (mA) & 100 & 10 & 1 \\
\hline Charge/b (nC) & 0.08 & 0.008 & 1.0 \\
\hline$\varepsilon_{x / y}(\mathrm{~nm})$ & 0.1 & 0.015 & 1 \\
\hline Energy (GeV) & 5.3 & 5.3 & 5.3 \\
\hline Rep. rate $(\mathrm{GHz})$ & 1.3 & 1.3 & 0.001 \\
\hline 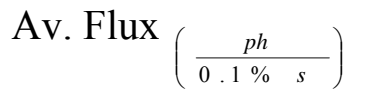 & $910^{15}$ & $910^{14}$ & $910^{12}$ \\
\hline Av. Brilliance & $1.610^{22}$ & $3.010^{22}$ & $2.010^{17}$ \\
\hline $\begin{array}{c}\overline{0.1 \% \mathrm{smm}_{\mathrm{mm}}^{2} \text { mrad }}{ }^{2} \\
\text { Bunch length }(\mathrm{ps})\end{array}$ & 2 & 2 & 0.1 \\
\hline
\end{tabular}


Table 2. Electron Beam Parameters of R\&D ERL

\begin{tabular}{|c|c|c|}
\hline Mode of operation & $\begin{array}{l}\text { High } \\
\text { charge }\end{array}$ & $\begin{array}{l}\text { High } \\
\text { current }\end{array}$ \\
\hline Initial bunch length, ps & 70 & 45 \\
\hline Initial beam radius, $\mathrm{mm}$ & 4.0 & 2.5 \\
\hline Field on the cathode $\mathrm{MeV} / \mathrm{m}$ & 44.2 & 27.8 \\
\hline Injection energy, $\mathrm{MeV}$ & 3.7 & 2.5 \\
\hline Maximum beam energy, $\mathrm{MeV}$ & 21 & 20 \\
\hline Average beam current, A & 0.2 & 0.5 \\
\hline Bunch rep-rate, $\mathrm{MHz}$ & 10 & 350 \\
\hline Charge per bunch, $\mathrm{nC}$ & 10 & 1.4 \\
\hline \multicolumn{3}{|l|}{ @ $2.5 \mathrm{MeV}$} \\
\hline Normalized emittances $(\mathrm{x} / \mathrm{y}), \mathrm{mm} * \mathrm{mrad}$ & $13.4 / 9.2$ & $4.1 / 4.1$ \\
\hline $\begin{array}{l}\text { Longitudinal emittance (with/without } 3^{\text {rd }} \\
\text { harmonic RF), psec*keV }\end{array}$ & $62 / 240$ & $19 / 58$ \\
\hline \multicolumn{3}{|l|}{ @ $20 \mathrm{MeV}$} \\
\hline Normalized emittances $(\mathrm{x} / \mathrm{y}), \mathrm{mm} * \mathrm{mrad}$ & $14.5 / 7.8$ & $2.5 / 1.3$ \\
\hline $\begin{array}{l}\text { Longitudinal projected }{ }^{(*)} \text { emittance } \\
\text { (with/without } 3^{\text {rd }} \text { harmonic RF). psec*keV }\end{array}$ & $183 / 1140$ & $42 / 700$ \\
\hline
\end{tabular}

${ }^{(*)}$ Note - real value of the longitudinal emittance remains very small while RMS value is determined by the curvature of the accelerating field 
Table 3. Main parameters of electron beam in the eRHIC

\begin{tabular}{|c|c|c|}
\hline Mode of operation & Collider & Light Source \\
\hline Circumference $[\mathrm{m}]$ & 3834 & 3834 \\
\hline Beam rep-rate & 28.15 & 703.75 \\
\hline Beam energy $[\mathrm{GeV}]$ & $5-20$ & $5-20$ \\
\hline$\gamma$, Relativistic factor & $1-410^{4}$ & $1-410^{4}$ \\
\hline RMS normalized emittance $[\mu \mathrm{m}]$ & $5-50$ & 0.9 \\
\hline Beam emittance @20 GeV [Å] & $1.25-12.5$ & 0.18 \\
\hline Full transverse coherence at $\lambda,[\AA$ & & 1.13 \\
\hline or at photon energy $[\mathrm{keV}]$ & & 11 \\
\hline RMS Bunch length [psec] & 30 & $0.03-3$ \\
\hline Charge per bunch $[\mathrm{nC}]$ & $1.6-16$ & 0.7 \\
\hline Average e-beam current $[\mathrm{A}]$ & $0.045-0.45$ & 0.5 \\
\hline
\end{tabular}

